\title{
Novel insights on gut microbiota manipulation and immune checkpoint inhibition in cancer (Review)
}

\author{
SILVIA VIVARELLI ${ }^{1}$, LUCA FALZONE $^{2}$, GIULIA COSTANZA LEONARDI ${ }^{1}$, \\ MARIO SALMERI ${ }^{1,3^{*}}$ and MASSIMO LIBRA ${ }^{1,3^{*}}$ \\ ${ }^{1}$ Department of Biomedical and Biotechnological Sciences, University of Catania, I-95123 Catania; \\ ${ }^{2}$ Epidemiology and Biostatistics Unit, IRCCS Istituto Nazionale Tumori 'Fondazione G. Pascale', I-80131 Naples; \\ ${ }^{3}$ Research Centre for Prevention, Diagnosis and Treatment of Cancer, University of Catania, I-95123 Catania, Italy
}

Received May 10, 2021; Accepted June 22, 2021

DOI: $10.3892 /$ ijo.2021.5255

\begin{abstract}
Cancer affects millions of individuals worldwide. Thus, there is an increased need for the development of novel effective therapeutic approaches. Tumorigenesis is often coupled with immunosuppression which defeats the anticancer immune defense mechanisms activated by the host. Novel anticancer therapies based on the use of immune checkpoint inhibitors (ICIs) are very promising against both solid and hematological tumors, although still exhibiting heterogeneous efficacy, as well as tolerability. Such a differential response seems to derive from individual diversity, including the gut microbiota (GM) composition of specific patients. Experimental evidence supports the key role played by the GM in the activation of the immune system response against malignancies. This observation suggests to aim for patient-tailored complementary therapies able to modulate the GM, enabling the selective enrichment in microbial species, which can improve the positive outcome of ICI-based immunotherapy. Moreover, the research of GM-derived predictive biomarkers may help to identify the selected cancer population, which can benefit from ICI-based therapy, without the occurrence of adverse reactions and/or cancer relapse. The present review summarizes the landmark studies published to date, which have contributed to uncovering the tight link existing between GM composition, cancer development and the host immune
\end{abstract}

Correspondence to: Dr Luca Falzone, Epidemiology and Biostatistics Unit, IRCCS Istituto Nazionale Tumori 'Fondazione G. Pascale', Via Mariano Semmola 53, I-80131 Naples, Italy

E-mail: 1.falzone@istitutotumori.na.it

Professor Massimo Libra, Department of Biomedical and Biotechnological Sciences, University of Catania, Via Santa Sofia 97, I-95123 Catania, Italy

E-mail: m.libra@unict.it

${ }^{*}$ Contributed equally

Key words: gut microbiome, dysbiosis, cancer, immunotherapy, immune checkpoint inhibitors, integrated therapy system. Bridging this triangle of interactions may ultimately guide towards the identification of novel biomarkers, as well as integrated and patient-tailored anticancer approaches with greater efficacy.

\section{Contents}

1. Introduction

2. Gut microbiota and the host immune system

3. Gut microbiota and cancer

4. Gut microbiota and immune checkpoint inhibition

5. Conclusions

\section{Introduction}

The gut microbiota (GM) is composed of $>100$ trillion of microorganisms (including bacteria, viruses, protozoa and fungi) resident in the gastro-intestinal lumen, mainly the large intestine (1). Among all, bacteria represent the broader category, with thousands of different species, belonging to the Firmicutes and Bacteroidetes phyla in particular (2). Gut microbes have been widely studied given their key role in the modulation of both the host homeostasis and pathology (3). The main functions of the GM include the following: i) The maintenance of the host's gut health; ii) gastrointestinal barrier function; and iii) the neo-synthesis or transformation of dietary compounds and essential nutrients (4-6). The fulfillment of all the aforementioned activities suggests the establishment of a functional two-way association between GM and the host immune system (7). This interrelation guarantees the preservation of a microbial balance or eubiosis (7). However, the absence of such an equilibrium (with the concurrent depletion of the gut microflora) is termed dysbiosis and is associated with a number of pathologies, including diabetes, inflammation, autoimmune disorders and cancer (8).

In order to examine the impact of GM on human health, it is important to characterize what is known as the gut microbiome, corresponding to the entire genome of the whole GM. The gut microbiome accounts for 100-fold more genes than the entire human genome (9). Presently, the advent of 
metagenomics allows the transition from merely depicting the microbiome composition to functionally analyzing the impact of the microbiome balance vs. imbalance in human health (10-12). The computational analysis of the 16S rRNA amplicons coupled with next-generation sequencing (NGS) allows the characterization of both the abundance and diversity of the gut microbiome. Overall, metagenomics, together with metatranscriptomics, metabolomics and proteomics help to quantify the impact of specific bacterial species on human health (13-15).

The reconstruction of the whole bacterial genomes beginning from metagenomic datasets is currently helping to identify uncharacterized bacterial species, both from the gut and other body sites, thus expanding the known phylogenetic diversity. The recent study from Almeida et al (16) established the ultimately most comprehensive collection of microbial genomes composing the human gut microbiome, comprised of $>200,000$ of non-redundant genomes from 4,644 gut prokaryotes. This will allow their use as a reference in future metagenomics studies (16). Given the tight interconnection occurring between the gut microbiome and the human host, it is of pivotal importance to identify all the host-dependent variables (e.g., physiology, lifestyle habits and diet), which alter the GM to further increase both robustness and the reproducibility of metadata analyses (17). This will help to identify the members of the GM that are directly associated with human diseases, including cancer, and ultimately with the response to anticancer therapy (7).

Cancer is a leading cause of mortality worldwide, second only to cardiovascular diseases, accounting for almost 10 million deaths in 2020 (18). Over the past 10 years, anticancer immunotherapy has taken the central stage in the treatment of a variety of tumors (19). In general, immunotherapy targets immune cells in order to activate or boost their capacity of eliminating cancerous cells. Malignant cells are surrounded by a variety non-cancer cells, including stromal cells and immune cells [e.g., macrophages, dendritic cells (DCs), natural killer (NK) cells, T-cells and B-cells], together forming the so-called tumor microenvironment $(20,21)$. The immune contexture' of a given tumor plays a key role in both the prognosis and treatment of cancer patients. Despite the presence of cancer immune-evasion mechanisms, any residual anticancer immune response may suggest a better prognosis. Moreover, anticancer therapies triggering the 'immune contexture' may produce a more durable anticancer efficacy (22).

Among the immunotherapies which are currently tested in clinical practice, immune checkpoint inhibitors (ICIs) have been shown to efficiently reshape the host immune response against cancer (23). The discovery of immune checkpoints led to the designation of the 2018 Nobel Prize in Physiology or Medicine to the two scientists, James Allison and Tasuku Honjo (24).

ICIs are monoclonal antibodies designed to inhibit the immune checkpoint pathway, thereby boosting the host immune system to efficiently eliminate cancer cells (25). In detail, ICIs trigger cytotoxic $\mathrm{CD}^{+} \mathrm{T}$-cells to destroy target malignant cells, thereby reactivating the cancer immunity cycle (26). In fact, the immune checkpoints are co-receptors of the $\mathrm{T}$ receptor signaling complex, which overall prevent $\mathrm{T}$-cell overactivation and establish the tolerance against self-antigens (27). The suppression of T-cell activation via these co-receptors constitutes the main immune escape mechanism carried out by neoplastic cells. In fact, when cancer cells efficiently activate the immune checkpoint, they may evade the immune system and overgrow (28). However, the administration of ICIs blocks these inhibitory co-receptors and positively modulates $\mathrm{CD} 8^{+} \mathrm{T}$-cell cytotoxicity, directing it to effectively eliminating malignant cells (28).

Approved ICIs can target two main co-inhibitory routes: Either the programmed cell death protein 1 (PD-1) or the cytotoxic T-lymphocyte antigen-4 (CTLA-4) pathway (29). PD-1 is expressed principally by T-cells and other immune cells (including NK cells, DCs and B-cells), whereas its ligand, programmed cell death ligand 1 (PD-L1) is expressed by the antigen-presenting cells (APCs), including malignant cells (30). Thus, antibodies directed against PD-1 or PD-L1 block the inhibitory immune checkpoint interaction between $\mathrm{CD}^{+} \mathrm{T}$-cells and tumor cells, rehabilitating cytotoxic T-cells to efficiently eliminate target cancer cells (31). Instead, CTLA4 is a receptor expressed by $\mathrm{T}-$ and $\mathrm{B}$-cells, and inhibits the binding of CD28 receptor with its B7 ligand, expressed by APCs at the earlier phases of antigen presentation (32). Consequently, ICIs targeting CTLA4 receptor reactivate the cellular-mediated immune response earlier in the cancer immunity cycle (33).

Since the first ICI was authorized by the US Food and Drug Administration (FDA) in 2011, a wide range of ICIs has been further approved (34). ICIs have been presently employed for the treatment of $\sim 50$ cancer types either as late-line, first-line, or as neoadjuvant therapies. They are administered either as single agents or in combination (with chemotherapy or with another ICI) (34). ICIs are currently under study in $>60 \%$ of all ongoing oncology clinical trials (for some examples on the specific use of ICIs in oncology in relation to the gut microbiome, please see the studies listed in Tables I and II) $(35,36)$. Impressively, for a number of recalcitrant and otherwise uncurable tumors, such as advanced melanoma (AM), metastatic melanoma (MM) or advanced non-small cell lung cancer (NSCLC), the use of ICIs has led to a substantial long-term remission $(37,38)$.

Despite this substantial clinical success, the administration of ICIs is accompanied by some limitations. Amongst the reported issues, there is the modification of the main clinical endpoints due to the often-associated delay in the appearance of positive effects mediated by ICI-based immunotherapy $(21,39)$. Additionally, in the majority of cases, patients with advanced disease finally develop resistance to ICIs, mainly due to the development of innate and adaptive immune-resistance to the checkpoint blockade $(40,41)$. Finally, ICI blockage may be associated with the occurrence of a broad range of immune-related adverse events (irAEs), caused by the potential immune and pro-inflammatory overactivation of the host's immune system. The reported irAEs are several, and include: Colitis, intestinal mucositis, diarrhea, thyroiditis, hepatitis, dermatological manifestations, pneumonitis, myocarditis and others. The outcome of irAEs can range from mild to severe and in some cases, fatal events occur $(42,43)$.

The differential response of patients observed with the use of ICIs, in terms of both efficacy and tolerability, can be linked to the intrinsic individual diversity of the immune system and other host-related factors (44). Thus, identifying effective 
Table I. Current ongoing clinical trials registered at clinicaltrials.gov that analyze the intestinal microbiota through metagenomics during immune-checkpoint immunotherapy (alone or in combination with other anti-cancer therapies).

\begin{tabular}{|c|c|c|c|c|c|}
\hline Tim & Condition(s) & Anticancer therapy & Enrollment & Start date & (Refs.) \\
\hline NCT02600143 & Melanoma & ICIs & 123 & 2013 & n.a. \\
\hline NCT01896999 & Hodgkin lymphoma & $\begin{array}{l}\text { Ipilimumab; nivolumab; } \\
\text { brentuximab }\end{array}$ & 126 & 2014 & $(146)$ \\
\hline NCT02478099 & Advanced solid tumors & MPDL3280A & 98 & 2016 & $(147)$ \\
\hline NCT02681302 & Multiple myeloma; lymphoma & Ipilimumab; nivolumab & 42 & 2016 & n.a. \\
\hline NCT04204434 & Advanced solid tumors & ICIs & 150 & 2016 & n.a. \\
\hline NCT02858921 & Melanoma & $\begin{array}{l}\text { Dabrafenib; trametinib; } \\
\text { pembrolizumab }\end{array}$ & 60 & 2017 & n.a. \\
\hline NCT03083691 & Non-small cell lung cancer & Ipilimumab; nivolumab & 106 & 2017 & n.a. \\
\hline NCT03161756 & Melanoma & $\begin{array}{l}\text { Ipilimumab; nivolumab; } \\
\text { denosumab }\end{array}$ & 72 & 2017 & n.a. \\
\hline NCT03164993 & Breast cancer & $\begin{array}{l}\text { Atezolizumab; doxorubicin; } \\
\text { cyclophosphamide }\end{array}$ & 75 & 2017 & $(148)$ \\
\hline NCT03168464 & Non-small cell lung cancer & $\begin{array}{l}\text { Ipilimumab; nivolumab; } \\
\text { radiotherapy }\end{array}$ & 45 & 2017 & n.a. \\
\hline NCT03331562 & Pancreatic cancer & Pembrolizumab & 24 & 2017 & n.a. \\
\hline NCT03289819 & Breast cancer & $\begin{array}{l}\text { Pembrolizumab; paclitaxel; } \\
\text { epirubicin; cyclophosphamide }\end{array}$ & 50 & 2018 & n.a. \\
\hline NCT03688347 & Lung cancer & ICIs & 60 & 2018 & n.a. \\
\hline NCT04054908 & Gastrointestinal cancer & SOC, ICIs & 60 & 2018 & n.a. \\
\hline NCT04169867 & Melanoma & $\begin{array}{l}\text { Nivolumab; ipilimumab; } \\
\text { atezolizumab }\end{array}$ & 1160 & 2018 & n.a. \\
\hline NCT04579978 & Advanced solid tumors & ICIs & 60 & 2018 & n.a. \\
\hline NCT03694834 & Endometrial cancer & Pembrolizumab & 20 & 2019 & n.a. \\
\hline NCT03799744 & Head and neck cancer & VCN-01; durvalumab & 20 & 2019 & n.a. \\
\hline NCT03818061 & Head and neck cancer & Atezolizumab; bevacizumab & 110 & 2019 & n.a. \\
\hline NCT03894007 & Breast cancer & $\begin{array}{l}\text { Docetaxel; carboplatin; } \\
\text { trastuzumab; pertuzumab; } \\
\text { epirubicin; cyclophosphamide; } \\
\text { atezolizumab }\end{array}$ & 190 & 2019 & n.a. \\
\hline NCT04006262 & Oeso-gastric cancer & Ipilimumab; nivolumab & 32 & 2019 & $(149)$ \\
\hline NCT04013542 & Lung cancer & $\begin{array}{l}\text { Ipilimumab; nivolumab; } \\
\text { radiotherapy }\end{array}$ & 20 & 2019 & n.a. \\
\hline NCT04133948 & Melanoma & $\begin{array}{l}\text { Nivolumab; ipilimumab; } \\
\text { domatinostat }\end{array}$ & 45 & 2019 & n.a. \\
\hline NCT04136470 & $\begin{array}{l}\text { Non-small cell lung cancer; } \\
\text { melanoma }\end{array}$ & ICIs & 130 & 2019 & n.a. \\
\hline NCT04196465 & $\begin{array}{l}\text { Gastric cancer; esophageal } \\
\text { cancer; liver cancer }\end{array}$ & IMC-001 & 48 & 2019 & n.a. \\
\hline NCT04291755 & $\begin{array}{l}\text { Non-small-cell lung cancer; } \\
\text { colorectal cancer }\end{array}$ & Pembrolizumab & 100 & 2019 & n.a. \\
\hline NCT03977571 & Renal cell cancer & Ipilimumab; Nivolumab & 400 & 2020 & n.a. \\
\hline NCT04063501 & Lung cancer & Anti-PD-1 antibodies & 80 & 2020 & n.a. \\
\hline NCT04090710 & Renal cell cancer & $\begin{array}{l}\text { Ipilimumab; nivolumab; } \\
\text { radiotherapy }\end{array}$ & 78 & 2020 & n.a. \\
\hline NCT04107168 & $\begin{array}{l}\text { Melanoma; renal cancer; } \\
\text { lung cancer }\end{array}$ & $\begin{array}{l}\text { Nivolumab; pembrolizumab; } \\
\text { ipilimumab; durvalumab; } \\
\text { tremelimumab; atezolizumab; } \\
\text { bevacizumab }\end{array}$ & 1800 & 2020 & n.a. \\
\hline NCT04189679 & Non-small cell lung cancer & ICIs & 60 & 2020 & n.a. \\
\hline NCT04207086 & Melanoma & Pembrolizumab; lenvatinib & 20 & 2020 & n.a. \\
\hline
\end{tabular}


Table I. Continued.

\begin{tabular}{|c|c|c|c|c|c|}
\hline Tim & Condition(s) & Anticancer therapy & Enrollment & Start date & (Refs.) \\
\hline NCT04271384 & Non-small cell lung cancer & Nivolumab; SOC & 30 & 2020 & n.a. \\
\hline NCT04312308 & Non-small cell lung cancer & Atezolizumab & 100 & 2020 & n.a. \\
\hline NCT04333004 & $\begin{array}{l}\text { Non-small cell lung cancer } \\
\text { (brain metastases) }\end{array}$ & $\begin{array}{l}\text { Pembrolizumab; } \\
\text { chemotherapy }\end{array}$ & 40 & 2020 & n.a. \\
\hline NCT04392505 & Non-small cell lung cancer & Durvalumab & 100 & 2020 & n.a. \\
\hline NCT04435964 & $\begin{array}{l}\text { Melanoma; lung cancer; head } \\
\text { and neck cancer; urogenital } \\
\text { cancer; breast cancer }\end{array}$ & ICIs & 400 & 2020 & n.a. \\
\hline NCT04566029 & Urothelial cancer & SOC, ICIs & 40 & 2020 & n.a. \\
\hline NCT04636775 & Non-small cell lung cancer & ICIs & 46 & 2020 & n.a. \\
\hline NCT04638751 & $\begin{array}{l}\text { Non-small cell lung cancer; } \\
\text { colorectal cancer; triple negative } \\
\text { breast cancer; pancreas cancer }\end{array}$ & ICIs, chemotherapy & 4000 & 2020 & n.a. \\
\hline NCT04680377 & $\begin{array}{l}\text { Non-small cell lung; advanced } \\
\text { lung cancer }\end{array}$ & Durvalumab & 44 & 2020 & n.a. \\
\hline NCT04169074 & Head and neck cancer & Nivolumab; abemaciclib & 20 & 2021 & n.a. \\
\hline NCT04602078 & Urothelial cancer & $\begin{array}{l}\text { Atezolizumab; gemcitabine; } \\
\text { cisplatin }\end{array}$ & 66 & 2021 & n.a. \\
\hline NCT04698161 & $\begin{array}{l}\text { Non-Small cell lung cancer; } \\
\text { melanoma }\end{array}$ & ICIs & 50 & 2021 & n.a. \\
\hline NCT04711330 & Non-small cell lung cancer & Durvalumab & 126 & 2021 & n.a. \\
\hline NCT04743752 & Non-small cell lung cancer & ICIs & 200 & 2021 & n.a. \\
\hline NCT04804137 & $\begin{array}{l}\text { Non-small cell lung cancer; } \\
\text { metastatic lung cancer }\end{array}$ & ICIs & 80 & 2021 & n.a. \\
\hline
\end{tabular}

ICIs, immune checkpoint inhibitors; SOC, standard of care; n.a., not available.

methods with which to identify the specific features of the individual immune system and direct it to better respond to ICIs represents the current challenge of ICI research (44). As it will be largely discussed below, the GM is a master regulator of the immune system; therefore, it directly affects both the efficacy and toxicity of ICIs $(45,46)$ (Fig. 1).

Consequently, the GM can be used as a powerful source to identify novel diagnostic and prognostic microbial-derived biomarkers, as well as innovative therapeutic targets $(47,48)$. In fact, recent milestone findings have highlighted the presence of specific gut bacterial species which are able to improve both the compliance to, as well as the effectiveness of anticancer therapies, particularly ICIs (49). Notably, in 2021, for the first time, to the best of our knowledge, two groundbreaking studies demonstrated that fecal microbiota transplantation (FMT) can efficiently boost the anticancer efficacy of ICIs in patients with AM and MM (50,51).

The present review summarizes the up-to-date studies on the role played by the GM in modulating the host immune system, thus influencing both the safety and the outcome to ICI-based anticancer therapy in cancer patients. Current findings indicate that each individual cancer patient has a specific GM footprint. Research efforts are presently focusing on developing effective strategies which can be used to manipulate the GM in a patient-tailored manner, with the aims of: i) Improving the efficacy of ICIs; and ii) actively reducing the occurrence of irAEs linked with ICI administration.

Compared with the existing literature on this topic, the general aim of the present review was to provide a concise and complete overview of the milestone studies that have contributed to deciphering the complicated association between gut microbial health, the host immune system and ICI activity over the past decade. Overall, the hidden potential of treating cancer patients with a more holistic therapeutic approach is strongly emerging, through the administration of integrated therapies (e.g., ICIs combined with GM modulators) tailored around the features of each specific patient (including gut microbial composition and immune system reactivity).

\section{Gut microbiota and the host immune system}

A dynamic two-way association occurs between GM and the host immune system through the course of a lifetime (52). The GM plays a key role in both shaping and modulating the immune system. In turn, the immune system regulates the gut microbial balance and it helps to maintain a healthy gut homeostasis (53). Any imbalance in this association could contribute to the development of several pathological conditions, including immune-mediated disorders, as well as cancer (54). 


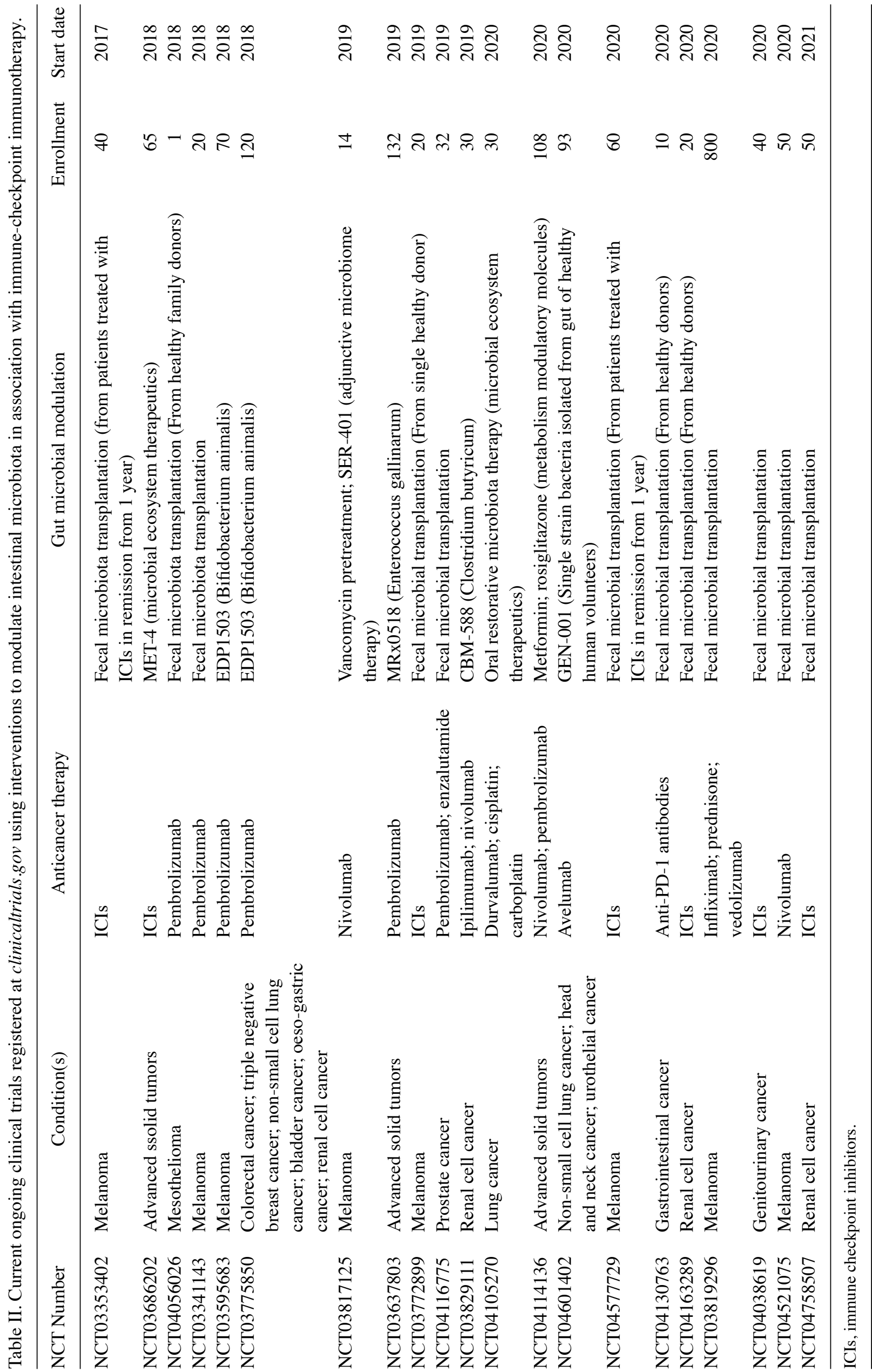




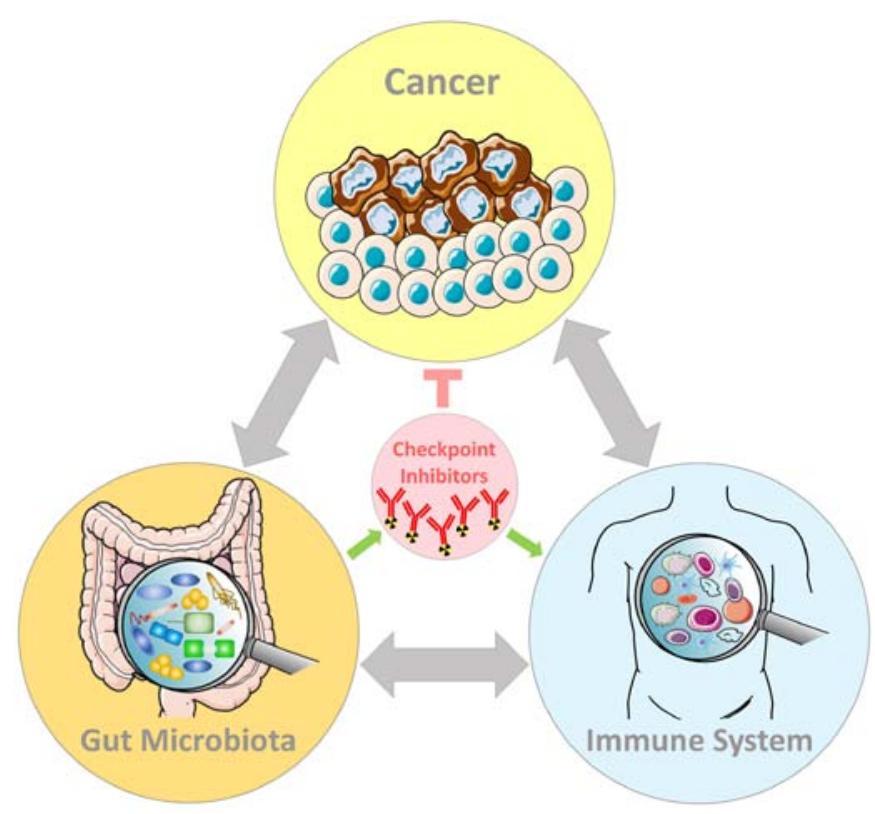

Figure 1. Complex interplay between gut microbiota, immune system and cancer during immune-checkpoint inhibition. Gut microbiota may positively or negatively modulate tumor growth; it may also regulate immune system response. Host immune system inhibits tumor growth and it can also be activated by gut microbiota. Cancer can alter host immune response by activating immunosuppressive pathways; also, cancer may modulate gut microbiota. Anticancer immunotherapy, based on the administration of immune checkpoint inhibitory antibodies, blocks tumor growth as it is able to inhibit immunosuppressive pathways. A favorable gut microbiota can increase immune checkpoint efficacy and moderate immune-adverse related reactions.

As regards innate immunity, since the very early years of life, the GM composition is actively shaped by the immune system and, in turn, the GM affects the development of the immune system (55). It has been demonstrated that since the maternal acquisition of the gut microbes during childbirth, critical interactions between the GM and the immune system may determine the establishment of both a eubiotic GM and a fully-functional immune activity (56). Any imbalance in the GM composition due, for example, to antibiotic-mediated depletion, may determine the instauration of immune-related diseases which can appear later in the adult life (such as asthma, or inflammatory bowel disease) $(57,58)$.

The intestinal mucosal barrier represents the interface between the gut microbiota and the human body (59). Below the mucosal layer resides the gut lumen which is composed of the following: i) Intestinal epithelial cells; ii) enteroendocrine cells; and iii) intraepithelial lymphocytes and other immune cells (60). This conserved structure allows the interaction of the commensal GM with the host immune system, together forming the so-called gut-immune axis (54). Although the immune system in the intestine evolves to fight invading pathogens, there is a delicate balance which allows the development of tolerance to non-dangerous commensals, as well as food antigens, although it fights pathogenic microbes that may otherwise invade the gut lumen and trespass the gut barrier (61).

At the gut lumen, intestinal goblet cells secrete high levels of hyperglycosylated mucin able to compartmentalize gut microbes within the mucosal surface and distant from the epithelial surface (62). Moreover, the glycans bound to mucin deliver tolerogenic signals, inducing intestinal local DCs to switch towards an anti-inflammatory state (62). Such DCs, once they internalize commensal microbes and express their antigens at the cell surface, selectively induce resident plasma cells to secrete $\operatorname{IgA}$ and protect the host from microbial invasion (63). In addition, Paneth cells of the small intestine secrete a range of antimicrobial peptides (AMPs), which restrain the expansion of potential microbial pathogens and hence help to maintain the GM homeostasis (64).

Pathogen-associated molecular patterns (PAMPs) comprise all the microbial-derived molecules, produced from both pathogens and commensals (65). PAMPs are actively recognized by pattern recognition receptors (PRRs), which are expressed by gut epithelial cells and local immune myeloid cells, and represent the main innate immune recognition pathway (65). PRRs are constantly exposed to PAMPs, also during gut homeostasis, in the absence of any infection (66).

PAMPs produced by gut commensals usually do not elicit a pro-inflammatory response. The specific context determines the outcome upon PRR activation (67). Only in the presence of epithelial damage, PAMPs enter the cytosolic cellular epithelial compartment (68). The elicited inflammatory response determines the activation of $\mathrm{NF}-\kappa \mathrm{B}$ signaling, further promoting the local secretion of pro-inflammatory cytokines, such as interferons (IFNs) (69). This pro-inflammatory response actively protects the intestine against microbial infections. On the contrary, in the absence of concurrent epithelial damage, PRR activation can be beneficial and may promote immune tolerance (70).

Similar to the innate response, the adaptive immune response is modulated in a two-way manner by GM, both locally and systemically (71). For instance, gut-associated B-cells secrete several grams of IgA per day within the gut lumen (72). This secretion can be either T-cell-independent or T-cell-dependent (72). Recently, it was also reported that gut mesenchymal cells can induce plasma cells to secrete IgA (73). In general, Foxp $3^{+}$regulatory T-cells (Tregs) induce a diversified $\operatorname{Ig} \mathrm{A}$ repertoire, which in turn maintains a heterogeneous and eubiotic GM. In turn, the healthy GM sustains the homeostatic $\operatorname{Ig} \mathrm{A}$ responses in a positive feedback loop (74). IgA in the lumen coat pathogenic bacteria, preventing their potential invasion and a subsequent pro-inflammatory response (75).

T-cells play a pivotal role in regulating both local and systemic adaptive immunity related to GM homeostasis, as well as pathology. GM and GM-derived molecules can induce $\mathrm{CD}^{+}{ }^{+} \mathrm{T}$-cell differentiation towards the main types: Th1, Th2, Tregs and Th17 (76). Th1 cells are essential against intracellular pathogens. Th2 cells are necessary during parasite-mediated infection. Tregs and Th17 are cellular phenotypes involved in the containment of the immune response. In particular, Tregs regulate the instauration of the immune tolerance (77). It has been observed that polysaccharide A (PSA) produced by Bacteroides fragilis induces the Th1 phenotype, while segmented filamentous bacteria (SFB) potently trigger Th17 differentiation $(78,79)$.

Th17 cells play a major role in mucosal immunity as they are able to prevent pathogen infection within the lamina propria, by secreting cytokines, including IL-17. IL-17 induces 
intestinal epithelial cells to express tight junctions and to secrete AMPs (80). Moreover, IL-17 further stimulates the release of other pro-inflammatory cytokines by neutrophils, which can be recruited from the main bloodstream and directed towards the gut (81).

Additionally, GM-mediated immune-cell priming can shape the systemic immune response. When APCs, such as DCs, present their antigens within the mesenteric lymph nodes to Tregs and Th17 cells, these T-cells can travel through the bloodstream and promote distal immune responses against cross-reacting antigens located in other sites of the body $(82,83)$. As a consequence, dysbiosis may affect systemic immune functions, thus increasing the susceptibility to certain infections and, for example, altering the response to vaccines (84).

Commensal gut microbes can actively secrete de novo produced molecules or transform the host's metabolites, which all may be sensed by nearby gut epithelial cells (85). Such bioproducts may have profound effects on the health of a host, including: i) Inducing immune-mediated protection against microbial pathogens; ii) maintaining gut barrier integrity; iii) metabolizing xenobiotics; iv) modulating the host's metabolism; and v) shaping and activating/inactivating the host immune system (86-89).

The GM is involved in the production of essential micronutrients, including vitamins $\mathrm{K}$ and B (90). In addition, a number of gut commensals can convert certain amino acids into signaling molecules, such as glutamate into gamma-aminobutyric acid (GABA) or histidine into histamine (90). Importantly, gut bacteria ferment dietary fibers to obtain a class of hormone-like bioproducts known as short-chain fatty acids (SCFAs), with multiple known functions in human health (91). For instance, SCFAs are transported to the liver representing an energy source. Additionally, SCFAs may control both glucose and lipid metabolism through the modulation of peptide hormone secretion by gut epithelial cells (92). Moreover, SCFAs (including butyrate) can enhance immunity, triggering the production of IgA by plasma cells (93). In turn, IgA inhibits bacterial adhesion to gut epithelial cells, hence blocking invasion $(94,95)$. Moreover, SCFAs can interfere with the balance between anti-inflammatory and pro-inflammatory cytokines secreted by immune cells, both locally and systemically. The consequence is the modulation of homeostatic Treg vs. the Th17 cell ratio, resulting in an immune imbalance $(96,97)$.

In summary, the preservation of a fine gut microbial equilibrium (in terms of the presence and relative abundance of commensal species) is imperative for sustaining and accomplish all the vital immune functions of the host. A healthy GM positively influences the immune system both locally and systemically. Conversely, once activated, the immune system may alter the GM balance. Gut eubiosis is thus of utmost importance for the maintenance of immune health (Fig. 2).

Notably, there is increasing evidence to indicate that gut dysbiosis may specifically affect local and systemic anti-tumor immunity. In fact, recurrent antibiotic exposure (which can impair intestinal eubiosis and favor the expansion of gut pathogens) is directly associated with an increased risk of cancer (98). In general, dysbiosis may influence tumor formation or ICI-based therapy failure (99). As largely depicted in the present review, a healthy (both enriched and diverse)
GM can activate the immune system to: i) Fight cancer; and ii) efficiently respond to anticancer immunotherapies, in particular ICIs (100).

\section{Gut microbiota and cancer}

Cancer is a multifactorial disease resulting from a combination of intrinsic factors (e.g., the stochastic accumulation of gene mutations and epigenetic alterations), environmental exposures (e.g., pollution, sunlight exposure and infections) and lifestyle habits (cigarette smoking, diet and sport) $(101,102)$. The resulting overall risk of developing a given malignancy is mainly dependent on the dose, duration, as well as the combination of exposures, all coupled with the specific genetic and epigenetic background (103).

Presently, several biological agents are listed as carcinogens by the International Agency of Research on Cancer (IARC), including a number of viruses (i.e., Epstein-Barr virus, hepatitis B virus, hepatitis C virus, Kaposi's sarcoma-associated herpesvirus, human immunodeficiency virus-1, human papillomaviruses and human T-cell lymphotropic virus type 1), as well as the gastrointestinal bacterial pathogen, Helicobacter pylori (104). Individuals with Helicobacter pylori infection have a higher risk of developing stomach cancer as the infection directly causes chronic inflammation (105).

Generally, the influence of gastrointestinal microorganisms on cancer development is complex (106). In fact, the GM plays a dual role in cancer, as gut microbes can either positively or negatively affect tumorigenesis, depending on their nature (49). Bacteria per se or their products may directly or indirectly affect tumorigenesis. In general, beneficial bacteria which are normally part of an eubiotic GM exert an antitumor effect (107). On the contrary, pathogens prevailing in a dysbiotic GM are pro-tumorigenic, either directly or through the production of microbial-derived toxins. These effects have been documented in both local colorectal cancers (CRCs), as well as in distant tumors (108). The authors recently reviewed all the relevant findings regarding the dual role played by the GM in cancer, focusing on the specific gut microorganisms involved (49).

Several preclinical findings have demonstrated the pro-tumorigenic role of a number of gut microbes. For instance, a number of bacteria, mostly pathogens, can release toxins within the intestinal lumen which, once internalized by lumen epithelial cells, can directly promote genotoxic damage or, alternatively, they can activate pro-proliferative or anti-apoptotic pathways (49). For example, cytolethal distending toxins (CDTs), Shiga-like and Shiga toxins secreted respectively by Escherichia coli, Helicobacter spp., Shigella dysenteriae, as well as several others, may directly induce DNA damage (109). Moreover, the surface molecule FadA from Fusobacterium nucleatum, CagL from Helicobacter pylori and SopB from Salmonella typhimurium can trigger the WNT/B-catenin, MEK-ERK and STAT3 pathways, respectively, all inducing target cells to over-proliferate and/or not undergo apoptosis (110-112).

Additionally, the GM may alter the immune response activated by the host against the neoplasm, as observed in several tumor-bearing mice models $(49,113,114)$. For example, enterotoxigenic Bacteroides fragilis infection in a mouse model of 


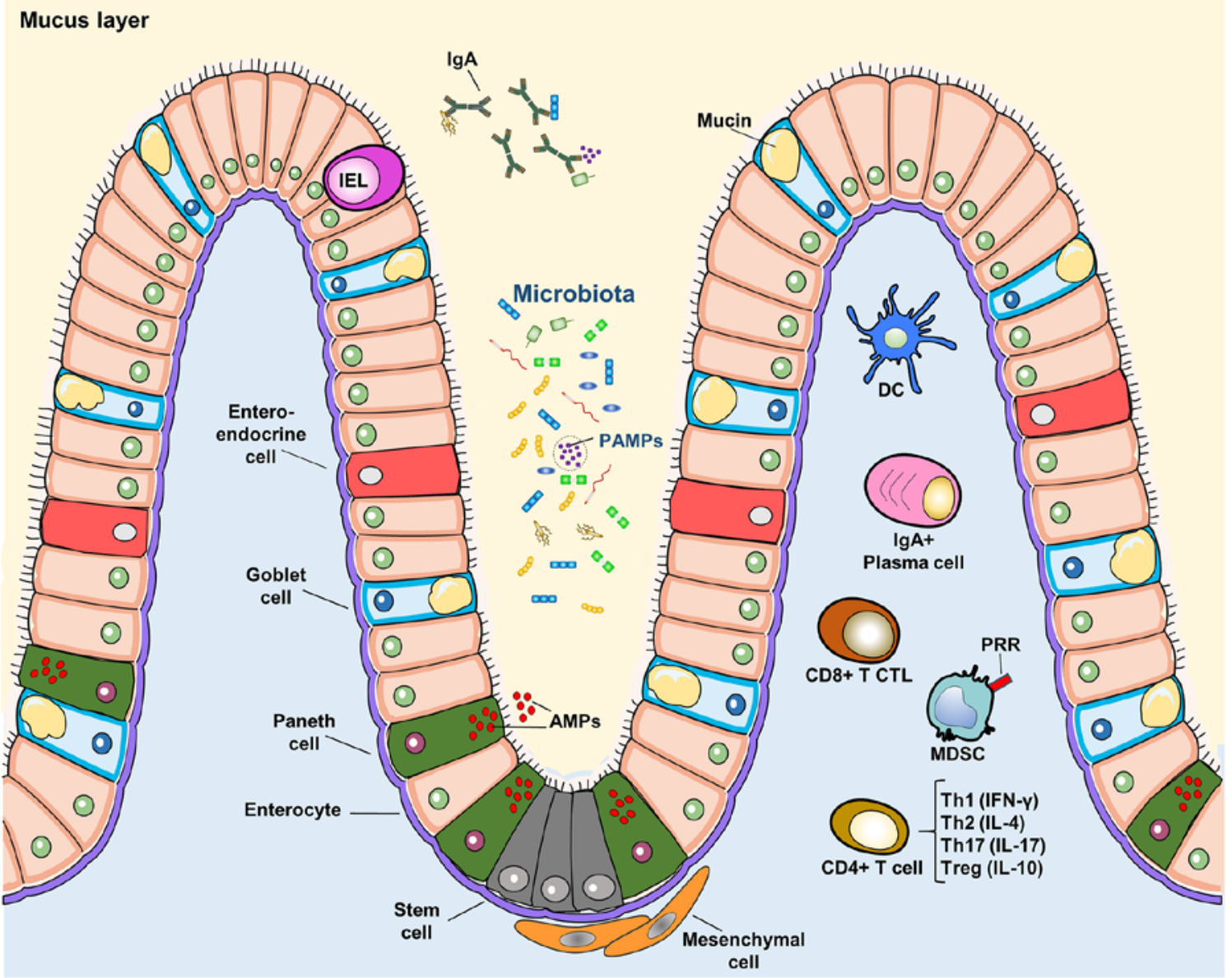

Figure 2. The gut-immune axis. The gastrointestinal lumen represents the interface between the GM and immune system. Intestinal cells constitute the villi structure and include: Enteroendocrine cells, goblet cells, Paneth cells, enterocytes and stem cells. Paneth cells secrete AMPs. IELs may reside within the epithelial structure. Goblet cells secrete mucin which enriches the intraluminal mucus layer. Gut microbiota and their derived molecules form PAMPs. PAMPs are recognized by PRRs expressed by immune cells and gut epithelial cells. IgA are secreted in the lumen and help to bind microbes and microbial-deriving molecules. Immune cells are pivotal in the instauration of the immune-tolerance versus commensals and immune-reactivity against pathogens. Both innate and adaptive immunity are involved. Immune cells include: DCs, IgA-producing plasma cells, CD8+ CTLs, MDSCs and CD4+ T-cells. The latter can differentiate into different phenotypes involved in immune reactivity or tolerance (i.e., Th1, Th2, Th17 and Tregs). GM, gut microbiota; AMPs, anti-microbial peptides; IELs, intestinal intraepithelial lymphocytes; PAMPs, pathogen-associated molecular patterns; PRRs, pattern recognition receptors; DCs, dendritic cells; CTLs, cytotoxic T lymphocytes; MDSCs, myeloid-derived suppressor cells.

$\mathrm{CRC}$ attracts a colonic immune infiltrate. In particular, it has been demonstrated that Bacteroides-derived enterotoxin BFT promotes the differentiation of pro-tumoral myeloid-derived suppressor cells (MDSC), which in turn produce NO and suppress $\mathrm{CD}^{+} \mathrm{T}$-cell proliferation $(115,116)$. In addition, the Treg response upon enterotoxigenic Bacteroides fragilis infection in mice with CRC triggers IL-17 and induces Th17 development which in turn, promotes tumorigenesis (117). Furthermore, Fusobacterium nucleatum has been shown to potentiate CRC tumorigenesis in a mouse model of CRC, driving MDSC infiltration within the intestinal tumors and inducing the activation of a general pro-tumoral immune-milieu (118).

By contrast, some bacteria have been shown to possess an anticancer function through the stimulation of the immune system of their host. For example, Bifidobacterium spp. promotes antitumor immunity in tumor-bearing mice through the activation of DCs, which enhance cytotoxic $\mathrm{CD}^{+}$T-cell activity directed against tumor cells (119). Akkermansia muciniphila and Enterococcus hirae orally administered to tumor-bearing mice have been shown to induce DCs to secrete IL-12, thus triggering the recruitment of $\mathrm{CD}^{+}$cytotoxic T-cells and inhibiting tumor growth (120) (Fig. 3).

Given all the aforementioned preclinical examples, it is clear that the maintenance of a healthy GM through the life of an individual may represent a good strategy with which to prevent cancer. A number of groundbreaking studies (described in detail below) have further expanded this concept, demonstrating that GM contains diagnostic and prognostic cancer biomarkers, suitable to identify therapy-responder patients. More importantly, either the modulation of the GM towards a healthy phenotype or the selective enrichment of GM in specific beneficial bacterial types may represent an enforcement to anticancer therapy. In this context, different studies have demonstrated how the administration of specific probiotic strains may positively influence the GM with beneficial effects for cancer patients and human health $(121,122)$. This concept particularly applies to immunomodulatory treatments and specifically to ICIs. 


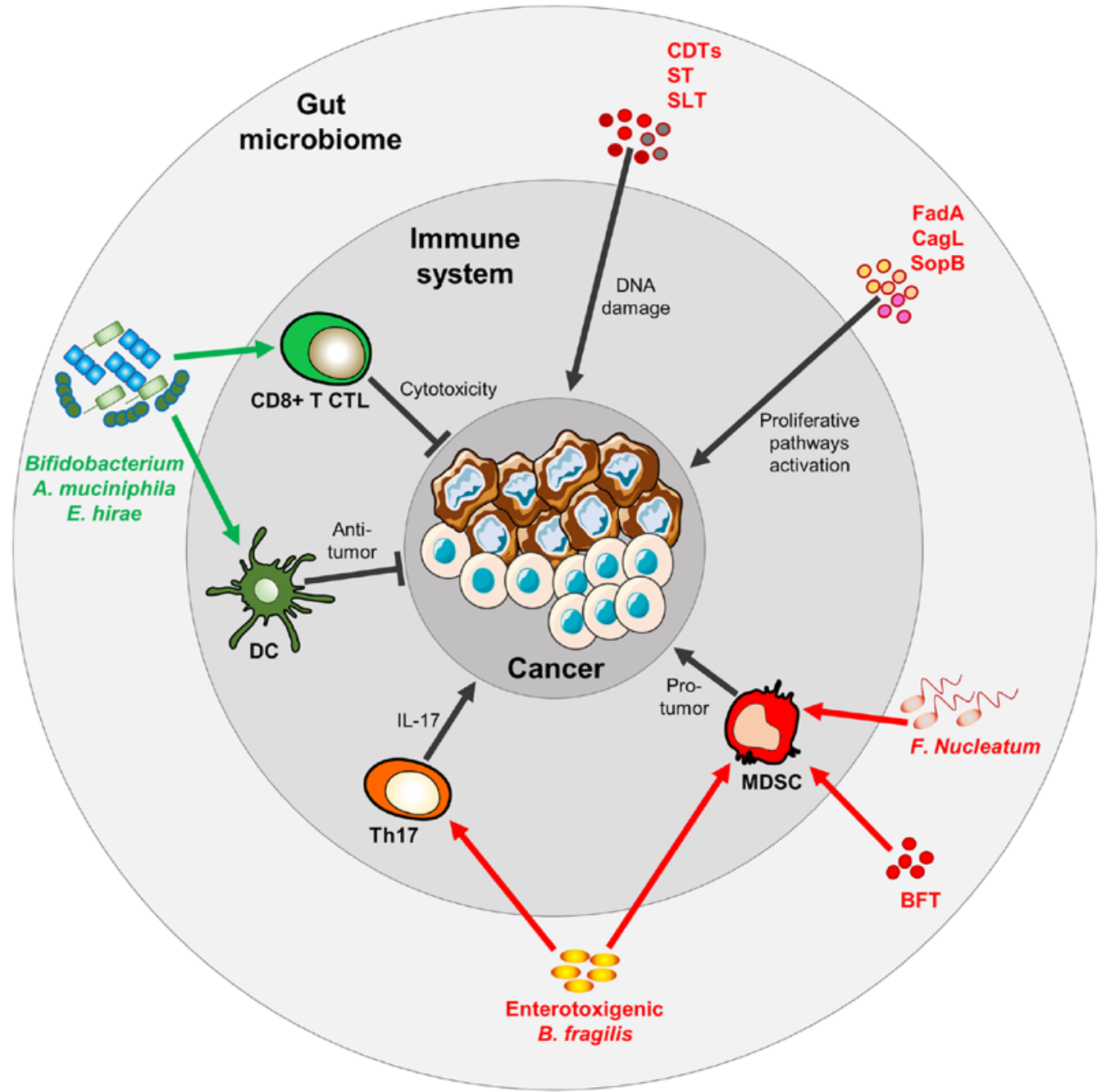

Figure 3. Gut microbiota play a dual role in cancer. Microbial-derived molecules, including CDTs, SLTs and STs may directly induce DNA damage and trigger cancer mutation. Other microbial surface molecules, such as FadA from Fusobacterium nucleatum, CagL from Helicobacter pylori and SopB from Salmonella typhimurium induce cancer cell proliferation. Fusobacterium nucleatum, Bacteroides fragilis or its derived Bacteroides fragilis toxin (BFT) can boost MDSCs which, in turn, favor a pro-tumoral milieu. Bacteroides fragilis may also trigger the Th17 T-cell phenotype, which is immunosuppressive and hence, pro-tumorigenic. Bifidobacterium spp., Akkermansia muciniphila, Enterococcus hirae promote DC activation and CD $8^{+}$cytotoxic T-cell activation, both triggering an anti-tumor immune response. CDTs, cytolethal distending toxins; SLT, Shiga-like toxin; ST, Shiga toxin; MDSCs, myeloid-derived suppressor cells; DC, dendritic cell.

\section{Gut microbiota and immune checkpoint inhibition}

A growing number of studies have shed further light on the association between the safety and efficacy of ICI-based immunotherapy and GM features in cancer patients. In this section, the authors aim to provide a temporal timeline demonstrating the progress made and milestones accomplished in this field.

In 2015, Vétizou et al (123) observed for the first time that tumors in antibiotic-treated or in germ-free mice did not respond to anti-CTLA4 immunotherapy.Pivotally, they demonstrated that the antitumor effect of anti-CTLA4 was dependent on Bacteroides spp., and in particular on Bacteroides fragilis. In fact, a significant antitumor response was observed: i) With oral gavage of Bacteroides fragilis; or ii) with immunization with Bacteroides fragilis-derived polysaccharides; or iii) with the adoptive transfer of Bacteroides fragilis-specific in vitro activated T-cells (123). The authors of that study performed FMT in tumor-bearing mice treated with anti-CTLA4 antibody. Specifically, they employed stools from melanoma patient donors with fecal abundance of Bacteroides spp. Following FMT, mice-derived feces were found to be selectively enriched in Bacteroides fragilis. This feature was negatively associated with tumor size following the CTLA-4 blockade in recipient mice. Hence, anti-CTLA4 antibody treatment could actively modify the abundance of immunogenic Bacteroides spp. in the gut, which in turn affected ICI-anticancer efficacy (123).

In the same year, Sivan et al (119) compared the growth of melanoma in mice grown in different breading facilities, thus bearing different GM compositions. They demonstrated significant differences in melanoma growth, which reflected the different cancer-specific T-cell response. Pivotally, anti-PD-L1-non-responder mice, when orally receiving either feces obtained from responder mice or Bifidobacterium alone, augmented their response to anti-PD-L1 therapy (119). In particular, a significant reduction in tumor outgrowth coupled with an augmented DC activity, leading to increased $\mathrm{CD} 8^{+} \mathrm{T}$-cell priming and T-cell accumulation in the tumor microenvironment was observed (119). 
Both studies strongly suggested that manipulating the GM could enhance the antitumor efficacy of ICIs $(119,123)$. These important observations paved the way for subsequent translational observations. In 2016, Dubin et al published a prospective study aimed at analyzing GM features in patients with MM treated with ICIs and developing colitis (124). The use of anti-CTLA4 antibody in cancer patients is often associated with dysbiosis and with inflammatory colitis as irAEs (124). The authors associated the fecal microbial composition at the baseline with the one following colitis manifestation. Notably, they found that an increased representation of bacteria belonging to the Bacteroidetes phylum was associated with augmented resistance to the development of ICI-induced colitis. On the contrary, microbiome analysis confirmed that patients lacking genetic pathways involved in polyamine transport and B vitamin biosynthesis had an increased risk of developing colitis (124).

In 2017, Frankel et al (125), through the use of metagenomic shotgun sequencing coupled with metabolomic profiling, identified the specific footprint of GM associated with the efficacy of ICIs in patients with MM. Of the 39 patients with MM, only a group exhibited a response to ICIs (corresponding to $67 \%$ of ipilimumab plus nivolumab-, and $23 \%$ of pembrolizumab-treated subjects). Despite the specific ICI used, feces from ICI-responders were enriched for Bacteroides caccae (125). In particular, responders treated with a combination of anti-CTLA4 and anti-PD-L1 had a GM enriched in Faecalibacterium prausnitzii, Bacteroides thetaiotamicron and Holdemania filiformis. However, responders treated with anti-PD-1 antibody had a GM enriched in Dorea formicogenerans. Among all the GM obtained from responders, the metabolite resulting consistently enriched was anacardic acid (125).

Also in 2017, Chaput et al (126) performed a prospective analysis on the fecal microbiota composition in 26 patients with MM. The analysis revealed that at the baseline, prior to any anti-CTLA4 antibody infusion, patients whose baseline microbiota was driven by Bacteroides exhibited a longer progression-free survival (PFS) than patients whose baseline microbiota was driven by Faecalibacterium genus plus other Firmicutes. Additionally, baseline colitis-associated phylotypes were selectively associated with the presence of Firmicutes (126). Upon ICI treatment, patients with MM belonging to the Faecalibacterium-driven cluster who developed anti-CTLA4-induced colitis exhibited a significant increase in the $\mathrm{CD}^{+} \mathrm{T}$-cell population and, in particular, in $\mathrm{CD}^{+} \mathrm{T}$-cells expressing the $\mathrm{T}$-cell inducible T-cell COStimulator surface marker. This observation suggested that the baseline GM composition may represent an important determinant of the immune response in cancer patients, as well as of anti-CTLA-4 associated-colitis (126).

Overall, the aforementioned prospective studies have demonstrated the importance of combining metagenomics and metabolomics to study the GM in cancer patients. These studies clearly demonstrate that GM is affected by ICI treatments. Thus, a specific GM composition and/or metabolite enrichment may be predictive of a better prognosis.

In 2018, three landmark studies were published in Science, clearly demonstrating, for the first time, a direct association between GM composition and efficacy of ICI-based therapy $(120,127,128)$. In particular, Routy et al (120) examined the effects of FMT from NSCLC and renal cell carcinoma (RCC) ICI-responder and ICI-non-responder donors, in recipient epithelial (melanoma and sarcoma) tumor-bearing mice (either germ-free or antibiotic-treated). They found that FMT from responders significantly enhanced the efficacy of ICIs in reducing tumor growth in mice, whereas FMT from non-responder patients did not exert any effect (120). Metagenomics analyses of fecal samples from responders and non-responders clearly revealed that the GM composition affected the primary immune-resistance to ICIs. In particular, a positive association was observed between the relative abundance of Akkermansia muciniphila and the clinical response to ICIs. The oral gavage of Akkermansia muciniphila in mice receiving FMT from non-responders significantly restored the efficacy of anti-PD-1 and augmented the recruitment of CD4 ${ }^{+}$ T-cells at the tumor site, and increased the local secretion of IL-12 by DCs (120).

Additionally, Matson et al (127) analyzed the baseline GM composition of stool samples from 42 patients with MM prior to receiving ICI therapy. Metagenomics analysis revealed that Bifidobacterium longum, Collinsella aerofaciens and Enterococcus faecium species were significantly enriched in ICI-responder patients (127). FMT from responder donors in recipient tumor-bearing mice improved the T-cell response and the anti-PD-L1 anticancer efficacy. In addition, from fecal analyses, 10 bacterial species were found to be differentially enriched in responder vs. non-responder mice. In total, eight of these (i.e., Enterococcus faecium, Collinsella aerofaciens, Bifidobacterium adolescentis, Klebsiella pneumoniae, Veillonella parvula, Parabacteroides merdae, Lactobacillus spp., and Bifidobacterium longum) were more abundant in responders, whereas two (i.e., Ruminococcus obeum and Roseburia intestinalis) were more abundant in non-responders (127).

Finally, Gopalakrishnan et al (128) analyzed the gut microbiome of 112 patients with MM, demonstrating that responders had a significantly higher alpha diversity and a relative abundance of bacteria belonging to the Ruminococcaceae family. Moreover, from metabolomics analyses, the authors of that study observed a significant enrichment of anabolic pathways. Responder patients also exhibited an enhanced systemic and antitumor immunity, with increased cytotoxic $\mathrm{CD}^{+} \mathrm{T}$-cell tumor infiltrates (128). Accordingly, germ-free mice receiving FMT from responders exhibited favorable immune profiling and an improved response to anti-PD-L1 antibody treatment in terms of tumor growth, which was significantly reduced. Importantly, gut microbiome analyses revealed that feces of responders were enriched in Clostridiales, whereas the feces of non-responders were rich in Bacteroidales (128).

Since these milestone studies, several others conducted on cancer patients have further revealed the existence of a relevant bacterial gut footprint in ICI-responders vs. non-responders. In 2018, Derosa et al (129) demonstrated how antibiotics can alter GM health, triggering antibiotic-associated dysbiosis. In turn, dysbiosis may invalidate the response to ICIs. In the retrospective analysis, patients with advanced RCC and NSCLC (121 and 239 patients, respectively) treated with anti-PD-L1 antibody (either as monotherapy or in combination) were considered. Above all, $13 \%$ of patients with RCC 
and $20 \%$ of patients with MM received antibiotics 30 days prior to the ICI administration. Of note, the antibiotics significantly reduced the benefits of ICIs in the cancer patients. In particular, PFS was significantly reduced in patients with RCC, whereas overall survival (OS) was decreased in patients with NSCLC (129).

In 2019, Jin et al (130) additionally confirmed that a favorable GM, as well as a healthy immune profile were associated with an improved response to anti-PD-1 immunotherapy. The authors considered 37 patients with advanced NSCLC. They performed the fecal microbiome analysis: i) At the moment of the anti-PD-1 therapy; ii) at the clinical evaluation; iii) following the progression of the disease. According to the Response Evaluation Criteria in Solid Tumor (RECST) scale, patients were divided in responders and non-responders (130). Responders which exhibited a significantly prolonged PFS had a high microbiome diversity. Compared with the baseline, the feces of responders were enriched in Alistipes putredinis, Bifidobacterium longum and Prevotella copri. However, non-responders exhibitd a prevalence in Ruminococcus spp. In addition, responders manifested an increase in $\mathrm{CD} 8^{+} \mathrm{T}$-cell and NK cell subsets in response to anti-PD-1 therapy (130).

Also in 2019, Zheng et al (131) performed gut microbiome profiling of a small cohort of patients with hepatocellular carcinoma (HCC), using metagenomic sequencing. Fecal samples from patients responding to anti-PD-1 immunotherapy exhibited a higher taxa richness and more gene counts than those of non-responders. Furthermore, dynamic sequencing analyses demonstrated that anti-PD-1 therapy increased the GM dissimilarity between responders and non-responders, with a prominence in such differences at 6 weeks post-treatment. A total of 20 responder-enriched species (including Akkermansia muciniphila and Ruminococcaceae spp.) were further identified as prominent. Subsequent metabolic pathway analysis demonstrated that carbohydrate metabolism and methanogenesis were selectively enriched in the responders (131).

Additionally, in 2020, Salgia et al (132) characterized the stool microbiome from 31 patients with metastatic RCC receiving ICIs (as single agents or combination) to assess treatment-related changes in GM composition over the course of treatment. They found that a higher microbial diversity was associated with better treatment outcomes. Temporal profiling of the microbiome indicated that the relative abundance of Akkermansia muciniphila significantly increased in patients obtaining clinical benefits from ICIs. Hence, dynamic changes in GM composition suggested the potential utility of modulating GM to reach more successful outcomes with ICIs (132).

Taken together, the clinical studies evidenced several microbial candidates, which can be suggested as predictive biomarkers of the patient population that may truly benefit from ICIs. Of equal importance is the elucidation of the molecular mechanisms responsible for the beneficial effect of the GM. For this purpose, several important preclinical studies have recently been published.

In 2019, Tanoue et al (114) isolated a consortium of 11 bacterial strains (comprised of seven Bacteroidales and four non-Bacteroidales species) from healthy human donor feces, capable of augmenting IFN- $\gamma$-secreting CD8 T-cell levels in the gut. The consortium, when inoculated into syngeneic CRC tumor-bearing germ-free mice, induced a robust MHCI expression in DCs and enhanced the therapeutic efficacy of ICIs, with a concurrent decrease in tumor growth (114).

In 2020, Xu et al (133) evaluated the effects of GM in MSS-type CRC tumor-bearing mice treated with different antibiotics prior to anti-PD-1 anticancer treatment. The injection of antibiotics significantly counteracted the efficacy of anti-PD-1 antibody in inhibiting tumor growth. Furthermore, metabolomics analysis demonstrated the enrichment in the glycerophospholipid metabolic pathway, specifically within the antibiotic-treated group. Changes in GM composition may drive these metabolic changes. In fact, Xu et al (133) demonstrated that Prevotella spp. and Akkermansia spp. were able to support the efficacy of anti-PD-1 by affecting the metabolism of glycerolipids in MSS-type CRC tumor-bearing mice.

Of note, in 2020, Mager et al (134) explored the underlying mechanisms through which the GM enhanced ICI-mediated antitumor immunity, with particular focus on the T-cell adaptive response. In detail, Bifidobacterium pseudolongum, Lactobacillus johnsonii and Olsenella spp. significantly increased the efficacy of ICIs in four different mouse models of cancer. In particular, Bifidobacterium pseudolongum modulated the ICI response through the production of the metabolite, inosine (134). They further assessed that a decreased gut barrier functionality induced by ICI-immunotherapy increased the systemic translocation of inosine, which in turn activated antitumor $\mathrm{CD}^{+} \mathrm{T}$-cell activity. Importantly, the effect of bacterial-derived inosine was strictly dependent on the T-cell surface expression of the adenosine A2A receptor, whose stimulation was specifically required (134).

More recently, in 2021, Si et al (135) evaluated the therapeutic efficacy of administering a single bacterial strain, Lactobacillus rhamnosus $G G$ (LGG) in combination with ICI immunotherapy. Pivotally, the oral administration of LGG significantly improved the efficacy of ICIs and reduced tumor growth in CRC and melanoma mouse models. The authors further explored the molecular mechanism, evidencing that the augmented anti-tumor activity of anti-PD-1 was associated with increased tumor-infiltrating DCs and CD8 ${ }^{+}$ T-cells (135). Moreover, treatments with live LGG alone or in combination with anti-PD-1 triggered type I IFN production by DCs, enhancing the cross-priming of $\mathrm{CD}^{+}$cytotoxic T-cells. In DCs, cyclic GMP-AMP synthase (cGAS)/stimulator of IFN genes (STING) was required for IFN- $\beta$ induction in response to LGG. In fact, LGG significantly boosted IFN- $\beta$ production via the cGAS/STING axis in DCs (135). The role of the STING pathway in potentiating the efficacy of immunotherapy, was also proven by Shi et al (136). In agreement with the findings of Si et al (135), Shi et al (136) observed that a specific gut microbe, Bifidobacterium spp., potentiated anti-CD47 immunotherapy via the stimulation of STING in DCs in tumor-bearing mice.

2021 was a breakthrough year in GM research associated with ICI therapeutic efficacy in human studies. In this year, two important publications in Science demonstrated, for the first time, that FMT significantly overcame the resistance to anti-PD-1 therapy in patients with MM $(50,51)$. Firstly, Davar et al (50) demonstrated that in patients with MM, the GM composition was associated with the anti-PD-1 response. 


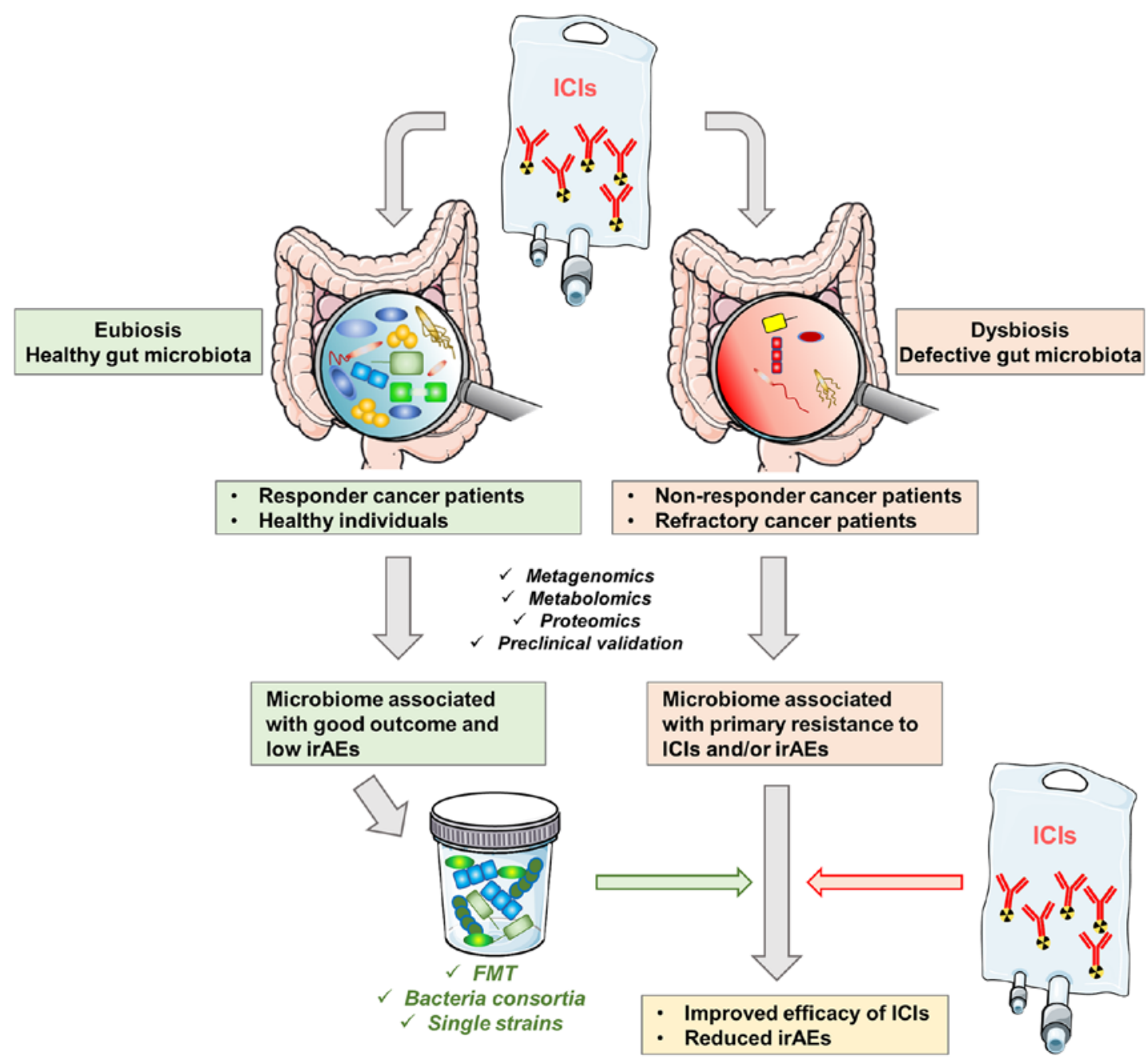

Figure 4. Gut microbiota as an adjuvant of immune checkpoint inhibition. ICIs affect the gut microbiota. In particular, responders have a eubiotic microbiota whereas non-responders have a dysbiotic and depauperated microbiota. Gut microbiota from responders or from healthy subjects is analyzed via metagenomics, metabolomics, proteomics and preclinical studies. This characterization allows to identify whole stools to perform a FMT or, alternatively, microbial consortia or single microbes that can be administered to non-responder cancer patients or cancer patients who (after an initial response) became refractory to ICIs. The therapeutic modulation of gut microbiota can be associated with ICIs to obtain an improved efficacy and/or a reduction in irAEs in refractory cancer patients. ICIs, immune checkpoint inhibitors; FMT, fecal microbiota transplantation; irAEs, immune-related adverse events.

Notably, the resistance to anti-PD-1 was overcome by directly modulating GM composition. The authors of that study evaluated both the safety and efficacy of responder-derived feces transplanted together with anti-PD-1 in recipient patients with PD-1-refractory melanoma (50). FMT with anti-PD-1 was well-tolerated and provided clinical benefit in 6 out of 15 patients. The combined treatment induced a rapid and robust gut microbiota perturbation. The six responders exhibited an increased abundance of taxa, such as Ruminococcaceae and Bifidobacteriaceae, that were previously shown to be associated with a response to anti-PD-1 associated with an increased $\mathrm{CD}^{+} \mathrm{T}$-cell activation, as well as with a decreased frequency of myeloid cells secreting IL-8 $(119,128)$. In addition, the responders exhibited distinct proteomic and metabolomic signatures. The trans-kingdom network analysis confirmed that the GM directly regulated these changes (50).

In parallel, Baruch et al (51) performed a pilot phase I clinical study to assess both the safety and feasibility of FMT and anti-PD-1 immunotherapy re-induction 10 patients with anti-PD-1-refractory MM. Notably, they observed a positive clinical response in 3 out of 10 patients (two partial responses and one complete response). Treatment with FMT was associated with favorable changes in immune cell infiltrates and immune-related gene expression profiles locally (at the level of the gut lamina propria), as well as distally (within the tumor microenvironment) (51).

Taken together, the reported findings provide strong evidence on the key role that the GM plays in modulating the ICI therapeutic response and potential-associated toxicity. Pivotally, Davar et al (50) and Baruch et al (51) demonstrated that gut microbial modulation can reverse ICI resistance in patients with MM through the specific modulation of the individual immune system, both locally and systemically (Fig. 4).

Since 2015, a number of clinical trials have been performed with two main goals. One important aim is to characterize the gut microbiome signature, as well as the associated immune system changes upon a specific ICI immunotherapy. The outcomes of these studies may reveal the existence of specific diagnostic and prognostic gut microbial biomarkers (Table I). 
Additionally, a growing number of clinical trials is currently evaluating both the safety and efficacy of actively modulating GM composition in association with ICI immunotherapy. Currently, a number of companies are investing in this direction and the number of registered trials is increasing exponentially (100). As presented in Table II, in order to 'turn bugs into drugs' a number of strategies are currently being tested, including the administration of: i) Single probiotics; ii) microbial communities; iii) synbiotics; iv) FMT; and v) metabolic modulators

Of note, for certain malignancies, such as prostate cancer, researchers have shown the limited efficacy of ICIs (137). This may be partially explained by the fact that prostate cancer is immunologically 'cold' compared to both melanoma and lung cancer, thus with a lower tumor mutational burden (TMB) $(138,139)$. Previous clinical studies have tested ICIs administered in combination with miscellaneous agents that may improve the overall efficacy of ICIs $(140,141)$. For instance, the concurrent administration of androgen inhibitors has been shown to significantly improve the OS of patients with metastatic castration-sensitive prostate cancer (142). Coherently, one ongoing trial reported (presented in Table II) is evaluating both the safety and efficacy of combining anti-PD-1 antibody with the anti-androgen enzalutamide and the FMT in prostate cancer patients not responding to initial ICI and androgen deprivation therapy. Prostate cancer patients with A significant initial response to anti-PD-1 and anti-androgens, will be used as fecal donors (NCT04116775).

\section{Conclusions}

Since birth, each individual inherits a specific GM footprint. Moreover, the GM is constantly shaped with age, diet and lifetime exposures. Current research has demonstrated the dual role played by the GM in cancer (49). In particular, the GM deeply affects the host immune system and vice versa. During dysbiosis, several microbial species can overpopulate the gut. Consequently, they may enhance inflammation and promote the formation of a pro-cancerogenic environment (143).

On the contrary, the re-establishment of a healthy GM may be beneficial for cancer patients, inducing a healthy immune system. In line with this observation, it has been further demonstrated that a specific ICI-responder gut microbial footprint is associated with a reduction in irAEs and an improvement of ICI-efficacy $(120,127,128)$. Furthermore, specific gut microbial modulatory therapy (based on FMT from healthy/responders donors) may revert ICI-resistance in patients with advanced cancer $(50,51)$. This latter groundbreaking observation is paving the way towards a growing number of ongoing clinical trials in cancer patients to test the administration of 'good' gut microbes as adjuvants in association with ICI-based therapy (Table II).

In spite of the tremendous progress made, some questions still remain unanswered. In fact, since cancer patients are often immunocompromised, special care needs to be taken to select the correct therapeutic strategy to modulate the GM, in order to maximize the positive outcomes of microbial-modulators, and reduce the potential harmful side-effects due to the possibly fragile immune system. For instance, a number of factors may affect the overall clinical outcome, including the use of concurrent medications such as antibiotics, which have been associated with a reduced response to ICIs (144). In addition, specific dietary associations are crucial and they can be efficiently used as predictors of FMT success, as demonstrated for patients with a higher intake of dietary fiber (145). The consumption of specific nutrients or bioactive food-derivatives may favor the FMT engraftment, particularly in cancer patients, who are often affected by nutritional and metabolic issues (e.g., vomiting, swallowing difficulties, reduced food adsorption and inadequate food consumption).

In conclusion, anticancer therapy is increasingly becoming holistic. In the near future, anticancer treatments will be tailored to the specific cancer patient, based on the GM if the individual, as well as the immune signature. Henceforward, larger clinical longitudinal studies will help to increase the current knowledge on the long-term safety and robustness of FMT as adjuvants of ICIs, in order to expand and standardize their use in a number of types of cancer.

\section{Acknowledgements}

The authors would like to thank the Italian League Against Cancer (LILT) for its support.

\section{Funding}

SV has been funded by a fellowship from the Italian League Against Cancer (LILT), Catania.

\section{Availability of data and materials}

Not applicable.

\section{Authors' contributions}

All authors (SV, LF, GCL, MS and ML) participated in the writing of the manuscript, and preparing the figures and tables, as well as in the revisions. SV and LF confirm the authenticity of all the raw data. All authors have read and approved the final manuscript.

\section{Ethics approval and consent to participate}

Not applicable.

\section{Patient consent for publication}

Not applicable.

\section{Competing interests}

ML is the principal investigator of a research grant founded by Dicofarm Spa to his University Department. In light of the fact that this manuscript is a Review of the existing literature, as well as of the ongoing clinical studies, the Dicofarm Spa will not benefit specifically from any of the findings described. The other authors declare that the research was conducted in the absence of any commercial or financial relationships that could be construed as a potential competing interest. 


\section{References}

1. Leviatan S and Segal E: Identifying gut microbes that affect human health. Nature 587: 373-374, 2020.

2. Greenhalgh K, Meyer KM, Aagaard KM and Wilmes P: The human gut microbiome in health: Establishment and resilience of microbiota over a lifetime. Environ Microbiol 18: 2103-2116, 2016.

3. Feng Q, Chen WD and Wang YD: Gut microbiota: An integral moderator in health and disease. Front Microbiol 9: 151, 2018.

4. Vaishnava S, Behrendt CL, Ismail AS, Eckmann L and Hooper LV: Paneth cells directly sense gut commensals and maintain homeostasis at the intestinal host-microbial interface. Proc Natl Acad Sci USA 105: 20858-20863, 2008.

5. Belkaid Y and Naik S: Compartmentalized and systemic control of tissue immunity by commensals. Nat Immunol 14: 646-653, 2013.

6. Magnúsdóttir S, Ravcheev D, de Crécy-Lagard V and Thiele I: Systematic genome assessment of B-vitamin biosynthesis suggests co-operation among gut microbes. Front Genet 6: 148 2015.

7. Zheng D, Liwinski $\mathrm{T}$ and Elinav E: Interaction between microbiota and immunity in health and disease. Cell Res 30: 492-506, 2020.

8. Sharma VR, Singh M, Kumar V, Yadav M, Sehrawat N, Sharma DK and Sharma AK: Microbiome dysbiosis in cancer: Exploring therapeutic strategies to counter the disease. Semin Cancer Biol 70: 61-70, 2021.

9. Sepich-Poore GD, Zitvogel L, Straussman R, Hasty J, Wargo JA and Knight R: The microbiome and human cancer. Science 371 eabc4552, 2021.

10. Integrative HMP (iHMP) Research Network Consortium: The integrative human microbiome project. Nature 569: 641-648, 2019.

11. Rothschild D, Weissbrod O, Barkan E, Kurilshikov A, Korem T, Zeevi D, Costea PI, Godneva A, Kalka IN, Bar N, et al: Environment dominates over host genetics in shaping human gut microbiota. Nature 555: 210-215, 2018.

12. Korem T, Zeevi D, Suez J, Weinberger A, Avnit-Sagi T, Pompan-Lotan M, Matot E, Jona G, Harmelin A, Cohen N, et al: Growth dynamics of gut microbiota in health and disease inferred from single metagenomic samples. Science 349 : 1101-1106, 2015.

13. Whon TW, Shin NR, Kim JY and Roh SW: Omics in gut microbiome analysis. J Microbiol 59: 292-297, 2021.

14. Geva-Zatorsky N, Sefik E, Kua L, Pasman L, Tan TG, Ortiz-Lopez A, Yanortsang TB, Yang L, Jupp R, Mathis D, et al: Mining the human gut microbiota for immunomodulatory organisms. Cell 168: 928-943.e11, 2017.

15. Haber AL, Biton M, Rogel N, Herbst RH, Shekhar K, Smillie C, Burgin G, Delorey TM, Howitt MR, Katz Y, et al: A single-cel survey of the small intestinal epithelium. Nature 551: 333-339, 2017.

16. Almeida A, Nayfach S, Boland M, Strozzi F, Beracochea M, Shi ZJ, Pollard KS, Sakharova E, Parks DH, Hugenholtz P, et al: A unified catalog of 204,938 reference genomes from the human gut microbiome. Nat Biotechnol 39: 105-114, 2021.

17. Vujkovic-Cvijin I, Sklar J, Jiang L, Natarajan L, Knight R and Belkaid Y: Host variables confound gut microbiota studies of human disease. Nature 587: 448-454, 2020.

18. Sung H, Ferlay J, Siegel RL, Laversanne M, Soerjomataram I, Jemal A and Bray F: Global cancer statistics 2020: GLOBOCAN estimates of incidence and mortality worldwide for 36 cancers in 185 countries. CA Cancer J Clin 71: 209-249, 2021.

19. Esfahani K, Roudaia L, Buhlaiga N, Del Rincon SV, Papneja N and Miller WH Jr: A review of cancer immunotherapy: From the past, to the present, to the future. Curr Oncol 27 (Suppl 2): S87-S97, 2020

20. Baghban R, Roshangar L, Jahanban-Esfahlan R, Seidi K, Ebrahimi-Kalan A, Jaymand M, Kolahian S, Javaheri T and Zare P: Tumor microenvironment complexity and therapeutic implications at a glance. Cell Commun Signal 18: 59, 2020.

21. Robert C: A decade of immune-checkpoint inhibitors in cancer therapy. Nat Commun 11: 3801, 2020.

22. Fridman WH, Zitvogel L, Sautès-Fridman C and Kroemer G The immune contexture in cancer prognosis and treatment. Nat Rev Clin Oncol 14: 717-734, 2017.

23. Hiam-Galvez KJ, Allen BM and Spitzer MH: Systemic immunity in cancer. Nat Rev Cancer 21: 345-359, 2021.
24. Ledford H, Else $\mathrm{H}$ and Warren M: Cancer immunologists scoop medicine nobel prize. Nature 562: 20-21, 2018.

25. Shin EC: Cancer immunotherapy: Special issue of BMB Reports in 2021. BMB Rep 54: 1, 2021.

26. Chen DS and Mellman I: Oncology meets immunology: The cancer-immunity cycle. Immunity 39: 1-10, 2013.

27. Huang PW and Chang JWC: Immune checkpoint inhibitors win the 2018 nobel prize. Biomed J 42: 299-306, 2019.

28. Waldman AD, Fritz JM and Lenardo MJ: A guide to cancer immunotherapy: From $\mathrm{T}$ cell basic science to clinical practice. Nat Rev Immunol 20: 651-668, 2020.

29. Shen CR and Chen YS: Immune checkpoint blockade therapy: The 2014 tang prize in biopharmaceutical science. Biomed J 38: $5-8,2015$

30. Topalian SL, Hodi FS, Brahmer JR, Gettinger SN, Smith DC, McDermott DF, Powderly JD, Carvajal RD, Sosman JA, Atkins MB, et al: Safety, activity, and immune correlates of anti-PD-1 antibody in cancer. N Engl J Med 366: 2443-2454, 2012.

31. Long J, Qi Z and Rongxin Z: PD-1/PD-L1 pathway blockade works as an effective and practical therapy for cancer immunotherapy. Cancer Biol Med 15: 116-123, 2018.

32. Seidel JA, Otsuka A and Kabashima K: Anti-PD-1 and anti-CTLA-4 therapies in cancer: Mechanisms of action, efficacy, and limitations. Front Oncol 8: 86, 2018.

33. Wei SC, Duffy CR and Allison JP: Fundamental mechanisms of immune checkpoint blockade therapy. Cancer Discov 8: 1069-1086, 2018.

34. Twomey JD and Zhang B: Cancer immunotherapy update: FDA-approved checkpoint inhibitors and companion diagnostics. AAPS J 23: 39, 2021.

35. Xin Yu J, Hubbard-Lucey VM and Tang J: Immuno-oncology drug development goes global. Nat Rev Drug Discov 18: 899-900, 2019.

36. Alsaab HO, Sau S, Alzhrani R, Tatiparti K, Bhise K, Kashaw SK and Iyer AK: PD-1 and PD-L1 checkpoint signaling inhibition for cancer immunotherapy: Mechanism, combinations, and clinical outcome. Front Pharmacol 8: 561, 2017.

37. Weber J, Mandala M, Del Vecchio M, Gogas HJ, Arance AM, Cowey CL, Dalle S, Schenker M, Chiarion-Sileni V, Marquez-Rodas I, et al: Adjuvant nivolumab versus ipilimumab in resected stage III or IV melanoma. N Engl J Med 377: 1824-1835, 2017

38. Eggermont AMM, Blank CU, Mandala M, Long GV, Atkinson V, Dalle S, Haydon A, Lichinitser M, Khattak A, Carlino MS, et al: Adjuvant pembrolizumab versus placebo in resected stage III melanoma. N Engl J Med 378: 1789-1801, 2018.

39. Kennedy LB and Salama AKS: A review of cancer immunotherapy toxicity. CA Cancer J Clin 70: 86-104, 2020.

40. Barrueto L, Caminero F, Cash L, Makris C, Lamichhane P and Deshmukh RR: Resistance to checkpoint inhibition in cancer immunotherapy. Transl Oncol 13: 100738, 2020.

41. Chen PL, Roh W, Reuben A, Cooper ZA, Spencer CN, Prieto PA, Miller JP, Bassett RL, Gopalakrishnan V, Wani K, et al: Analysis of immune signatures in longitudinal tumor samples yields insight into biomarkers of response and mechanisms of resistance to immune checkpoint blockade. Cancer Discov 6: 827-837, 2016.

42. Moslehi JJ, Salem JE, Sosman JA, Lebrun-Vignes B and Johnson DB: Increased reporting of fatal immune checkpoint inhibitor-associated myocarditis. Lancet 391: 933, 2018.

43. Teixidor E and Bosch-Barrera J: The dark side of immunotherapy: Challenges facing the new hope in cancer treatment. Ann Transl Med 7 (Suppl 6): S183, 2019.

44. Havel JJ, Chowell D and Chan TA: The evolving landscape of biomarkers for checkpoint inhibitor immunotherapy. Nat Rev Cancer 19: 133-150, 2019.

45. Pezo RC, Wong M and Martin A: Impact of the gut microbiota on immune checkpoint inhibitor-associated toxicities. Therap Adv Gastroenterol 12: 1756284819870911, 2019.

46. Chang AE, Golob JL, Schmidt TM, Peltier DC, Lao CD and Tewari M: Targeting the gut microbiome to mitigate immunotherapy-induced colitis in cancer. Trends Cancer 7: 583-593, 2021.

47. Sarshar M, Scribano D, Ambrosi C, Palamara AT and Masotti A: Fecal microRNAs as innovative biomarkers of intestinal diseases and effective players in host-microbiome interactions. Cancers (Basel) 12: 2174, 2020.

48. Murciano-Goroff YR, Warner AB and Wolchok JD: The future of cancer immunotherapy: Microenvironment-targeting combinations. Cell Res 30: 507-519, 2020. 
49. Vivarelli S, Salemi R, Candido S, Falzone L, Santagati M, Stefani S, Torino F, Banna GL, Tonini G and Libra M: Gut microbiota and cancer: From pathogenesis to therapy. Cancers (Basel) 11: 38, 2019

50. Davar D, Dzutsev AK, McCulloch JA, Rodrigues RR, Chauvin JM, Morrison RM, Deblasio RN, Menna C, Ding Q, Pagliano O, et al: Fecal microbiota transplant overcomes resistance to anti-PD-1 therapy in melanoma patients. Science 371 : 595-602, 2021

51. Baruch EN, Youngster I, Ben-Betzalel G, Ortenberg R, Lahat A, Katz L, Adler K, Dick-Necula D, Raskin S, Bloch N, et al: Fecal microbiota transplant promotes response in immunotherapy-refractory melanoma patients. Science 371: 602-609, 2021.

52. Maynard CL, Elson CO, Hatton RD and Weaver CT: Reciprocal interactions of the intestinal microbiota and immune system. Nature 489: 231-241, 2012.

53. Belkaid Y and Hand TW: Role of the microbiota in immunity and Inflammation. Cell 157: 121-141, 2014.

54. Jain T, Sharma P, Are AC, Vickers SM and Dudeja V: New insights into the cancer-microbiome-immune axis: Decrypting a decade of discoveries. Front Immunol 12: 622064, 2021.

55. Koenig JE, Spor A, Scalfone N, Fricker AD, Stombaugh J, Knight R, Angenent LT and Ley RE: Succession of microbial consortia in the developing infant gut microbiome. Proc Natl Acad Sci USA 108 (Suppl 1): S4578-S4585, 2011.

56. Gomez de Agüero M, Ganal-Vonarburg SC, Fuhrer T, Rupp S, Uchimura Y, Li H, Steinert A, Heikenwalder M, Hapfelmeier S Sauer U, et al: The maternal microbiota drives early postnatal innate immune development. Science 351: 1296-1302, 2016.

57. Russell SL, Gold MJ, Hartmann M, Willing BP, Thorson L, Wlodarska M, Gill N, Blanchet MR, Mohn WW, McNagny KM and Finlay BB: Early life antibiotic-driven changes in microbiota enhance susceptibility to allergic asthma. EMBO Rep 13: 440-447, 2012

58. Cahenzli J, Köller Y, Wyss M, Geuking MB and McCoy KD: Intestinal microbial diversity during early-life colonization shapes long-term IgE levels. Cell Host Microbe 14: 559-570, 2013.

59. Ge Y, Wang X, Guo Y, Yan J, Abuduwaili A, Aximujiang K, Yan J and Wu M: Gut microbiota influence tumor developmen and alter interactions with the human immune system. J Exp Clin Cancer Res 40: 42, 2021

60. Shi N, Li N, Duan X and Niu H: Interaction between the gut microbiome and mucosal immune system. Mil Med Res 4: 14, 2017.

61. Lazar V, Ditu LM, Pircalabioru GG, Gheorghe I, Curutiu C, Holban AM, Picu A, Petcu L and Chifiriuc MC: Aspects of gut microbiota and immune system interactions in infectious diseases, immunopathology, and cancer. Front Immunol 9: 1830, 2018.

62. Shan M, Gentile M, Yeiser JR, Walland AC, Bornstein VU, Chen K, He B, Cassis L, Bigas A, Cols M, et al: Mucus enhances gut homeostasis and oral tolerance by delivering immunoregulatory signals. Science 342: 447-453, 2013.

63. Macpherson AJ and Uhr T: Induction of protective IgA by intestinal dendritic cells carrying commensal bacteria. Science 303 1662-1665, 2004

64. Bevins CL and Salzman NH: Paneth cells, antimicrobial peptides and maintenance of intestinal homeostasis. Nat Rev Microbiol 9: 356-368, 2011

65. Chu $\mathrm{H}$ and Mazmanian SK: Innate immune recognition of the microbiota promotes host-microbial symbiosis. Nat Immunol 14: 668-675, 2013.

66. Vance RE, Isberg RR and Portnoy DA: Patterns of pathogenesis: Discrimination of pathogenic and nonpathogenic microbes by the innate immune system. Cell Host Microbe 6: 10-21, 2009.

67. Mogensen TH: Pathogen recognition and inflammatory signaling in innate immune defenses. Clin Microbiol Rev 22: 240-273, 2009.

68. Suresh R and Mosser DM: Pattern recognition receptors in innate immunity, host defense, and immunopathology. Adv Physiol Educ 37: 284-291, 2013.

69. Gaudet RG, Guo CX, Molinaro R, Kottwitz H, Rohde JR, Dangeard AS, Arrieumerlou C, Girardin SE and Gray-Owen SD Innate recognition of intracellular bacterial growth is driven by the TIFA-dependent cytosolic surveillance pathway. Cell Rep 19: 1418-1430, 2017

70. Bai L, Li W, Zheng W, Xu D, Chen N and Cui J: Promising targets based on pattern recognition receptors for cancer immunotherapy. Pharmacol Res 159: 105017, 2020.
71. Luis Muñoz-Carrillo J, Francisco Contreras-Cordero J, Gutiérrez-Coronado O, Trinidad Villalobos-Gutiérrez $\mathrm{P}$, Guillermo Ramos-Gracia L and Elizabeth Hernández-Reyes V: Cytokine profiling plays a crucial role in activating immune system to clear infectious pathogens. In: Immune Response Activation and Immunomodulation. IntechOpen, 2019. doi: 10.5772/intechopen.80843.

72. Kim M and Kim CH: Regulation of humoral immunity by gut microbial products. Gut Microbes 8: 392-399, 2017.

73. Nagashima K, Sawa S, Nitta T, Tsutsumi M, Okamura T, Penninger JM, Nakashima T and Takayanagi H: Identification of subepithelial mesenchymal cells that induce IgA and diversify gut microbiota. Nat Immunol 18: 675-682, 2017.

74. Cong Y, Feng T, Fujihashi K, Schoeb TR and Elson CO: A dominant, coordinated T regulatory cell- $\mathrm{IgA}$ response to the intestinal microbiota. Proc Natl Acad Sci USA 106: 19256-19261, 2009.

75. Tezuka $\mathrm{H}$ and Ohteki T: Regulation of IgA production by intestinal dendritic cells and related cells. Front Immunol 10: 1891, 2019.

76. $\mathrm{Wu} \mathrm{HJ}$ and $\mathrm{Wu} \mathrm{E}$ : The role of gut microbiota in immune homeostasis and autoimmunity. Gut Microbes 3: 4-14, 2012.

77. Lee GR: The Balance of Th17 versus Treg cells in autoimmunity. Int J Mol Sci 19: 730, 2018

78. Mazmanian SK, Liu CH, Tzianabos AO and Kasper DL: An immunomodulatory molecule of symbiotic bacteria directs maturation of the host immune system. Cell 122: 107-118, 2005.

79. Gaboriau-Routhiau V, Rakotobe S, Lécuyer E, Mulder I, Lan A, Bridonneau C, Rochet V, Pisi A, De Paepe M, Brandi G, et al: The key role of segmented filamentous bacteria in the coordinated maturation of gut helper $\mathrm{T}$ cell responses. Immunity 31 : 677-689, 2009

80. Valeri M and Raffatellu M: Cytokines IL-17 and IL-22 in the host response to infection. Pathog Dis 74: ftw111, 2016.

81. Liu R, Lauridsen HM, Amezquita RA, Pierce RW, Jane-Wit D, Fang C, Pellowe AS, Kirkiles-Smith NC, Gonzalez AL and Pober JS: IL-17 promotes neutrophil-mediated immunity by activating microvascular pericytes and not endothelium. J Immunol 197: 2400-2408, 2016.

82. Stary G, Olive A, Radovic-Moreno AF, Gondek D, Alvarez D, Basto PA, Perro M, Vrbanac VD, Tager AM, Shi J, et al: VACCINES. A mucosal vaccine against chlamydia trachomatis generates two waves of protective memory T cells. Science 348: aaa8205, 2015.

83. Hirota K, Duarte JH, Veldhoen M, Hornsby E, Li Y, Cua DJ, Ahlfors H, Wilhelm C, Tolaini M, Menzel U, et al: Fate mapping of IL-17-producing T cells in inflammatory responses. Nat Immunol 12: 255-263, 2011.

84. Oh JZ, Ravindran R, Chassaing B, Carvalho FA, Maddur MS, Bower M, Hakimpour P, Gill KP, Nakaya HI, Yarovinsky F, et al: TLR5-mediated sensing of gut microbiota is necessary for antibody responses to seasonal influenza vaccination. Immunity 41 : 478-492, 2014

85. Sandrini S, Aldriwesh M, Alruways M and Freestone P: Microbial endocrinology: Host-bacteria communication within the gut microbiome. J Endocrinol 225: R21-R34, 2015.

86. Gensollen T, Iyer SS, Kasper DL and Blumberg RS: How colonization by microbiota in early life shapes the immune system. Science 352: 539-544, 2016

87. Schmidt TSB, Raes J and Bork P: The human gut microbiome: From association to modulation. Cell 172: 1198-1215, 2018.

88. Bultman SJ: Emerging roles of the microbiome in cancer. Carcinogenesis 35: 249-255, 2014

89. Cani PD: Human gut microbiome: Hopes, threats and promises. Gut 67: 1716-1725, 2018

90. LeBlanc JG, Chain F, Martín R, Bermúdez-Humarán LG, Courau S and Langella P: Beneficial effects on host energy metabolism of short-chain fatty acids and vitamins produced by commensal and probiotic bacteria. Microb Cell Fact 16: 79, 2017.

91. Nakkarach A, Foo HL, Song AA, Mutalib NEA, Nitisinprasert S and Withayagiat $\mathrm{U}$ : Anti-cancer and anti-inflammatory effects elicited by short chain fatty acids produced by Escherichia coli isolated from healthy human gut microbiota. Microb Cell Fact 20: 36, 2021.

92. Clarke G, Stilling RM, Kennedy PJ, Stanton C, Cryan JF and Dinan TG: Minireview: Gut microbiota: The neglected endocrine organ. Mol Endocrinol 28: 1221-1238, 2014.

93. Pabst O: New concepts in the generation and functions of IgA. Nat Rev Immunol 12: 821-832, 2012

94. Mantis NJ, Rol N and Corthésy B: Secretory IgA's complex roles in immunity and mucosal homeostasis in the gut. Mucosal Immunol 4: 603-611, 2011. 
95. Mathias A, Pais B, Favre L, Benyacoub J and Corthésy B: Role of secretory IgA in the mucosal sensing of commensal bacteria. Gut Microbes 5: 688-695, 2014.

96. Levy M, Kolodziejczyk AA, Thaiss CA and Elinav E: Dysbiosis and the immune system. Nat Rev Immunol 17: 219-232, 2017.

97. Round JL and Mazmanian SK: Inducible Foxp3 ${ }^{+}$regulatory T-cell development by a commensal bacterium of the intestinal microbiota. Proc Natl Acad Sci USA 107: 12204-12209, 2010.

98. Cianci R, Franza L, Schinzari G, Rossi E, Ianiro G, Tortora G, Gasbarrini A, Gambassi G and Cammarota G: The interplay between immunity and microbiota at intestinal immunological niche: The case of cancer. Int J Mol Sci 20: 501, 2019.

99. Shui L, Yang X, Li J, Yi C, Sun Q and Zhu H: Gut microbiome as a potential factor for modulating resistance to cancer immunotherapy. Front Immunol 10: 2989, 2020

100.Zipkin M: Fecal microbiota potentiate checkpoint inhibitors, unleash microbiome startups. Nat Biotechnol 39: 529-532, 2021

101. Hanahan D and Weinberg RA: Hallmarks of cancer: The next generation. Cell 144: 646-674, 2011.

102. Niedzwiecki MM, Walker DI, Vermeulen R, Chadeau-Hyam M, Jones DP and Miller GW: The exposome: Molecules to populations. Annu Rev Pharmacol Toxicol 59: 107-127, 2019.

103. Arem H and Loftfield E: Cancer epidemiology: A survey of modifiable risk factors for prevention and survivorship. Am J Lifestyle Med 12: 200-210, 2017.

104.IARC Working Group on the Evaluation of Carcinogenic Risks to Humans: Biological agents. Volume 100 B. A review of human carcinogens. IARC Monogr Eval Carcinog Risks Hum 100: 1-441, 2012

105. Wroblewski LE, Peek RM Jr and Wilson KT: Helicobacter pylori and gastric cancer: Factors that modulate disease risk. Clin Microbiol Rev 23: 713-739, 2010.

106. Whisner CM and Athena Aktipis C: The role of the microbiome in cancer initiation and progression: How microbes and cancer cells utilize excess energy and promote one another's growth. Curr Nutr Rep 8: 42-51, 2019.

107. Villéger R, Lopès A, Carrier G, Veziant J, Billard E, Barnich N, Gagnière $\mathrm{J}$, Vazeille $\mathrm{E}$ and Bonnet $\mathrm{M}$ : Intestinal microbiota: A novel target to improve anti-tumor treatment? Int J Mol Sci 20 $4584,2019$.

108. Li W, Deng X and Chen T: Exploring the modulatory effects of gut microbiota in anti-cancer therapy. Front Oncol 11: 644454, 2021.

109. Guerra L, Cortes-Bratti X, Guidi R and Frisan T: The biology of the cytolethal distending toxins. Toxins (Basel) 3: 172-190, 2011.

110. Sun J, Hobert ME, Duan Y, Rao AS, He TC, Chang EB and Madara JL: Crosstalk between NF-kappaB and beta-catenin pathways in bacterial-colonized intestinal epithelial cells. Am J Physiol Gastrointest Liver Physiol 289: G129-G137, 2005.

111. Liu X, Lu R, Wu S and Sun J: Salmonella regulation of intestinal stem cells through the Wnt/beta-catenin pathway. FEBS Lett 584: 911-916, 2010.

112. Scanu T, Spaapen RM, Bakker JM, Pratap CB, Wu LE Hofland I, Broeks A, Shukla VK, Kumar M, Janssen H, et al: Salmonella manipulation of host signaling pathways provokes cellular transformation associated with gallbladder carcinoma. Cell Host Microbe 17: 763-774, 2015.

113. Li Y, Tinoco R, Elmén L, Segota I, Xian Y, Fujita Y, Sahu A, Zarecki R, Marie K, Feng Y, et al: Gut microbiota dependent anti-tumor immunity restricts melanoma growth in Rnf5 $5^{-1-}$ mice Nat Commun 10: 1492, 2019.

114. Tanoue T, Morita S, Plichta DR, Skelly AN, Suda W, Sugiura Y, Narushima S, Vlamakis H, Motoo I, Sugita K, et al: A defined commensal consortium elicits CD8 T cells and anti-cancer immunity. Nature 565: 600-605, 2019.

115. Valguarnera E and Wardenburg JB: Good gone bad: One toxin away from disease for Bacteroides fragilis. J Mol Biol 432: 765-785, 2020.

116. Thiele Orberg E, Fan H, Tam AJ, Dejea CM, Destefano Shields CE, Wu S, Chung L, Finard BB, Wu X, Fathi P, et al: The myeloid immune signature of enterotoxigenic Bacteroides fragilis-induced murine colon tumorigenesis. Mucosal Immunol 10: 421-433, 2017.

117. Geis AL, Fan H, Wu X, Wu S, Huso DL, Wolfe JL, Sears CL, Pardoll DM and Housseau F: Regulatory T-cell response to enterotoxigenic Bacteroides fragilis colonization triggers IL17-dependent colon carcinogenesis. Cancer Discov 5: 1098-1109, 2015.
118. Kostic AD, Chun E, Robertson L, Glickman JN, Gallini CA, Michaud M, Clancy TE, Chung DC, Lochhead P, Hold GL, et al: Fusobacterium nucleatum potentiates intestinal tumorigenesis and modulates the tumor-immune microenvironment. Cell Host Microbe 14: 207-215, 2013

119. Sivan A, Corrales L, Hubert N, Williams JB, Aquino-Michaels K, Earley ZM, Benyamin FW, Lei YM, Jabri B, Alegre ML, et al: Commensal Bifidobacterium promotes antitumor immunity and facilitates anti-PD-L1 efficacy. Science 350: 1084-1089, 2015.

120. Routy B, Le Chatelier E, Derosa L, Duong CPM, Alou MT, Daillère R, Fluckiger A, Messaoudene M, Rauber C, Roberti MP, et al: Gut microbiome influences efficacy of PD-1-based immunotherapy against epithelial tumors. Science 359: 91-97, 2018

121. Sharifi-Rad J, Rodrigues CF, Stojanović-Radić Z, Dimitrijević M, Aleksić A, Neffe-Skocińska K, Zielińska D, Kołożyn-Krajewska D, Salehi B, Milton Prabu S, et al: Probiotics: Versatile bioactive components in promoting human health. Medicina (Kaunas) 56: 433, 2020

122. Banna GL, Torino F, Marletta F, Santagati M, Salemi R, Cannarozzo E, Falzone L, Ferraù $\mathrm{F}$ and Libra $\mathrm{M}$ Lactobacillus rhamnosus GG: An overview to explore the rationale of its use in cancer. Front Pharmacol 8: 603, 2017.

123. Vétizou M, Pitt JM, Daillère R, Lepage P, Waldschmitt N, Flament C, Rusakiewicz S, Routy B, Roberti MP, Duong CP, et al: Anticancer immunotherapy by CTLA-4 blockade relies on the gut microbiota. Science 350: 1079-1084, 2015

124. Dubin K, Callahan MK, Ren B, Khanin R, Viale A, Ling L, No D, Gobourne A, Littmann E, Huttenhower C, et al: Intestinal microbiome analyses identify melanoma patients at risk for checkpoint-blockade-induced colitis. Nat Commun 7: 10391, 2016.

125. Frankel AE, Coughlin LA, Kim J, Froehlich TW, Xie Y, Frenkel EP and Koh AY: Metagenomic shotgun sequencing and unbiased metabolomic profiling identify specific human gut microbiota and metabolites associated with immune checkpoint therapy efficacy in melanoma patients. Neoplasia 19: 848-855, 2017.

126. Chaput N, Lepage P, Coutzac C, Soularue E, Le Roux K, Monot C, Boselli L, Routier E, Cassard L, Collins M, et al: Baseline gut microbiota predicts clinical response and colitis in metastatic melanoma patients treated with ipilimumab. Ann Oncol 28: 1368-1379, 2017

127. Matson V, Fessler J, Bao R, Chongsuwat T, Zha Y, Alegre ML, Luke JJ and Gajewski TF: The commensal microbiome is associated with anti-PD-1 efficacy in metastatic melanoma patients. Science 359: 104-108, 2018.

128. Gopalakrishnan V, Spencer CN, Nezi L, Reuben A, Andrews MC, Karpinets TV, Prieto PA, Vicente D, Hoffman K, Wei SC, et al: Gut microbiome modulates response to anti-PD-1 immunotherapy in melanoma patients. Science 359: 97-103, 2018.

129. Derosa L, Hellmann MD, Spaziano M, Halpenny D, Fidelle M, Rizvi H, Long N, Plodkowski AJ, Arbour KC, Chaft JE, et al: Negative association of antibiotics on clinical activity of immune checkpoint inhibitors in patients with advanced renal cell and non-small-cell lung cancer. Ann Oncol 29: 1437-1444, 2018

130. Jin Y, Dong H, Xia L, Yang Y, Zhu Y, Shen Y, Zheng H, Yao C, Wang Y and Lu S: The diversity of gut microbiome is associated with favorable responses to anti-programmed death 1 immunotherapy in chinese patients with NSCLC. J Thorac Oncol 14: 1378-1389, 2019.

131. Zheng Y, Wang T, Tu X, Huang Y, Zhang H, Tan D, Jiang W, Cai S, Zhao P, Song R, et al: Gut microbiome affects the response to anti-PD-1 immunotherapy in patients with hepatocellular carcinoma. J Immunother Cancer 7: 193, 2019.

132. Salgia NJ, Bergerot PG, Maia MC, Dizman N, Hsu J, Gillece JD, Folkerts M, Reining L, Trent J, Highlander SK and Pal SK: Stool microbiome profiling of patients with metastatic renal cell carcinoma receiving anti-PD-1 immune checkpoint inhibitors. Eur Urol 78: 498-502, 2020.

133. Xu X, Lv J, Guo F, Li J, Jia Y, Jiang D, Wang N, Zhang C, Kong L, Liu Y, et al: Gut Microbiome influences the efficacy of PD-1 antibody immunotherapy on MSS-type colorectal cancer via metabolic pathway. Front Microbiol 11: 814, 2020.

134. Mager LF, Burkhard R, Pett N, Cooke NCA, Brown K, Ramay H, Paik S, Stagg J, Groves RA, Gallo M, et al: Microbiome-derived inosine modulates response to checkpoint inhibitor immunotherapy. Science 369: 1481-1489, 2020. 
135. Si W, Liang H, Bugno J, Xu Q, Ding X, Yang K, Fu Y, Weichselbaum RR, Zhao X and Wang L: Lactobacillus rhamnosus GG induces cGAS/STING-dependent type I interferon and improves response to immune checkpoint blockade. Gut: gutjnl-2020-323426, 2021 (Epub ahead of print). doi: 10.1136/gutjnl-2020-323426.

136. Shi Y, Zheng W, Yang K, Harris KG, Ni K, Xue L, Lin W, Chang EB, Weichselbaum RR and Fu YX: Intratumoral accumulation of gut microbiota facilitates CD47-based immunotherapy via STING signaling. J Exp Med 217: e20192282, 2020.

137. Beer TM, Kwon ED, Drake CG, Fizazi K, Logothetis C, Gravis G, Ganju V, Polikoff J, Saad F, Humanski P, et al: Randomized, double-blind, phase III Trial of ipilimumab versus placebo in asymptomatic or minimally symptomatic patients with metastatic chemotherapy-naive castration-resistant prostate cancer. J Clin Oncol 35: 40-47, 2017

138. Berger MF, Lawrence MS, Demichelis F, Drier Y, Cibulskis K, Sivachenko AY, Sboner A, Esgueva R, Pflueger D, Sougnez C, et al: The genomic complexity of primary human prostate cancer. Nature 470: 214-220, 2011.

139. Nicholson LT and Fong L: Immune checkpoint inhibition in prostate cancer. Trends Cancer 6: 174-177, 2020.

140. Venkatachalam S, McFarland TR, Agarwal N and Swami U: Immune checkpoint inhibitors in prostate cancer. Cancers (Basel) 13: 2187, 2021.

141. Crocetto F, Boccellino M, Barone B, Di Zazzo E, Sciarra A, Galasso G, Settembre G, Quagliuolo L, Imbimbo C, Boffo S, et al: The crosstalk between prostate cancer and microbiota inflammation: Nutraceutical products are useful to balance this interplay? Nutrients 12: 2648, 2020.

142. Ferro M, Lucarelli G, Crocetto F, Dolce P, Verde A, La Civita E, Zappavigna S, de Cobelli O, Di Lorenzo G, Facchini BA, et al: First-line systemic therapy for metastatic castration-sensitive prostate cancer: An updated systematic review with novel findings. Crit Rev Oncol Hematol 157: 103198, 2021.

143. Barbosa AM, Gomes-Gonçalves A, Castro AG and Torrado E: Immune system efficiency in cancer and the microbiota influence. Pathobiology 88: 170-186, 2021.
144. Chen D, Wu J, Jin D, Wang B and Cao H: Fecal microbiota transplantation in cancer management: Current status and perspectives. Int J Cancer 145: 2021-2031, 2019.

145. Thompson S, Guetterman H, Taylor A, Bogner A, Martin D, Farrell JJ, Swanson KS and Holscher H: Dietary predictors of fecal microbiota transplantation success. J Acad Nutr Diet 116 (Suppl 9): A76, 2016.

146. Diefenbach CS, Hong F, Ambinder RF, Cohen JB, Robertson MJ, David KA, Advani RH, Fenske TS, Barta SK, Palmisiano ND, et al: Ipilimumab, nivolumab, and brentuximab vedotin combination therapies in patients with relapsed or refractory Hodgkin lymphoma: Phase 1 results of an open-label, multicentre, phase 1/2 trial. Lancet Haematol 7: e660-e670, 2020.

147. Bensch F, van der Veen EL, Lub-de Hooge MN, Jorritsma-Smit A, Boellaard R, Kok IC, Oosting SF, Schröder CP, Hiltermann TJN, van der Wekken AJ, et al: ${ }^{89} \mathrm{Zr}$-atezolizumab imaging as a non-invasive approach to assess clinical response to PD-L1 blockade in cancer. Nat Med 24: 1852-1858, 2018.

148. Kyte JA, Røssevold A, Falk RS and Naume B: ALICE: A randomized placebo-controlled phase II study evaluating atezolizumab combined with immunogenic chemotherapy in patients with metastatic triple-negative breast cancer. J Transl Med 18: 252, 2020

149. Cohen R, Pudlarz T, Garcia-Larnicol ML, Vernerey D, Dray X, Clavel L, Jary M, Piessen G, Zaanan A, Aparicio T, et al: Localized MSI/dMMR gastric cancer patients, perioperative immunotherapy instead of chemotherapy: The GERCOR NEONIPIGA phase II study is opened to recruitment. Bull Cancer 107: 438-446, 2020 (In French).

This work is licensed under a Creative Commons Attribution-NonCommercial-NoDerivatives 4.0 International (CC BY-NC-ND 4.0) License. 